\title{
Next-generation sequencing and the expanding domain of phylogeography
}

\section{Citation}

Edwards, S. V, A. Shultz, and S. C. Campbell-Staton. 2015. Next-generation sequencing and the expanding domain of phylogeography. Folia Zoologica 64 (3): 187-206.

\section{Published Version}

http://www.ivb.cz/folia-zoologica-archive.html?vol=64\&no=3

\section{Permanent link}

http://nrs.harvard.edu/urn-3:HUL.InstRepos:27716528

\section{Terms of Use}

This article was downloaded from Harvard University's DASH repository, and is made available under the terms and conditions applicable to Open Access Policy Articles, as set forth at http:// nrs.harvard.edu/urn-3:HUL.InstRepos:dash.current.terms-of-use\#OAP

\section{Share Your Story}

The Harvard community has made this article openly available.

Please share how this access benefits you. Submit a story.

Accessibility 


\title{
Next-generation sequencing and the expanding domain of phylogeography
}

\author{
Scott V. Edwards ${ }^{1 *}$, Allison J. Shultz ${ }^{1}$ and Shane C. Campbell-Staton ${ }^{1}$ \\ ${ }^{1}$ Department of Organismic and Evolutionary Biology, and Museum of Comparative Zoology, \\ Harvard University, Cambridge, MA 02138 USA
}

*Corresponding author:

Scott Edwards

Department of Organismic and Evolutionary Biology

Harvard University

Cambridge, MA 02138, USA

Email: sedwards@fas.harvard.edu 


\begin{abstract}
Phylogeography is experiencing a revolution brought on by next generation sequencing methods. A historical survey of the phylogeographic literature suggests that phylogeography typically incorporates new questions, expanding on its classical domain, when new technologies offer novel or increased numbers of molecular markers. A variety of methods for subsampling genomic variation, including Restriction site associated DNA sequencing (Rad-seq) and other next generation approaches, are proving exceptionally useful in helping define major phylogeographic lineages within species as well as details of historical demography. Next generation methods are also blurring the edges of phylogeography and related fields such as association mapping of loci under selection, and the emerging paradigm is one of simultaneously inferring both population history across geography and genomic targets of selection. However, recent examples, including some from our lab on Anolis lizards and songbirds, suggest that genome subsampling methods, while extremely powerful for the classical goals of phylogeography, may fail to allow phylogeography to fully achieve the goals of this new, expanded domain. Specifically, if genome-wide linkage disequilibrium is low, as is the case in many species with large population sizes, most genome subsampling methods will not sample densely enough to detect selected variants, or variants closely linked to them. We suggest that whole-genome resequencing methods will be essential for allowing phylogeographers to robustly identify loci involved in phenotypic divergence and speciation, while at the same time allowing free choice of molecular markers and further resolution of the demographic history of species.
\end{abstract}


Like many fields in evolutionary biology, phylogeography has been consistently

3 transformed by available technologies for assaying genetic variation. Indeed, the various

4 approaches for measuring genetic variation have waxed and waned as available technologies

5 come and go. A determinist viewpoint might suggest that major trends in marker use over the

6 decades have been driven largely by available technologies, such as PCR and next-generation

7 sequencing (reviewed in Brito and Edwards 2009). However, a more 'free will' perspective

8 might suggest that marker choice is also driven also by the conceptual needs of the discipline and

9 a rallying of the field behind core concepts that argue for one set of markers over another. What

10 seems clear is that, as technologies available to phylogeographers have changed, the borders of

11 the discipline - the types of questions and hypotheses that are tackled - also change. In

12 particular, phylogeography now appears to regularly pose questions that were traditionally the

13 domain of its closely allied sister discipline, population genetics, and increasingly in the domain

14 of finding the loci responsible for phenotypic variation in natural populations. These new

15 questions themselves can also drive the shape of the field and the tools that phylogeographers

16 adopt in order to answer them. In this essay we explore the history and future of phylogeography

17 through this lens of changing technologies and questions. We suggest that the domains of

18 phylogeography have expanded to include surveys of selection and covariation of genes and

19 environment across the landscape as a result of the increasing ability to assay variation at large

20 numbers of loci through next-generation sequencing. We suggest that trends in marker use imply

21 both a degree of technological determinism as well as shifts in free choice of markers over time.

22 Like other recent reviews, we foresee a day when a greatly expanded toolkit of markers and 
23 phylogeographic questions will be readily available through routine whole-genome resequencing

24 of geographically sampled populations.

\section{Molecular markers and the core concepts of phylogeography}

26 In the 1990s, the polymerase chain reaction (PCR) was the primary driver, and this led to

27 a proliferation of studies focusing on mitochondrial DNA in animals, and on chloroplast DNA in

28 plants. The focus on organellar genomes was not necessarily prescribed by PCR, but the ease of

29 amplification using PCR and the challenges of working routinely with nuclear genes perceived

30 by phylogeographers made organelle genomes a practical focus. MtDNA and cpDNA were also

31 attractive because they were polymorphic at the intraspecific level and experienced little if any

32 recombination, making it straightforward to move from DNA sequence to gene tree without

33 additional data manipulation. With this focus on organellar genomes came the oft-repeated

34 caveats now familiar to every student of phylogeography (Edwards and Bensch 2009): that

35 differences in gene flow between the sexes might cause organelle genomes to recover a biased

36 history of a given species; that the smaller effective population size of organellar genomes might

37 cause the genetic lineages to track population lineages more faithfully than the average marker,

38 sometimes resulting in an overly simplistic view of population history; and, more recently, that

39 natural selection on organelle genomes, particularly mtDNA, might yield estimates of genetic

40 diversity, or spatial patterns in the distribution of that diversity, which do not reflect neutral

41 processes or recent population history (Rand 2001; Excoffier and Ray 2008; Nabholz et al. 2008,

42 2009). Rather, lineage-specific mutation rates, which are hard to predict for a given species from

43 first principles or life history parameters, appear to be the best predictor of variation in genetic

44 diversity across species, at least in birds and mammals. Natural selection has also emerged as a

45 key determinant of mitochondrial diversity within species, as well as of relationships between 
46 mtDNA haplotype or codon distributions and latitude or thermal environment, potentially

47 compromising efforts to understand neutral diversity via neutral markers (Gerber et al. 2001;

48 Ballard and Whitlock 2004; Ribeiro et al. 2011; Jobling 2012; Ballard and Pichaud 2014;

49 Morales et al. 2015). Although genetic diversity within populations and species is not always a

50 primary focus of phylogeographic studies, it is certainly a basic descriptor of population history

51 and the forces governing it warrant our attention.

52 The first forays in to the nuclear genome in animal phylogeography came in the early

53 1990s in the form of PCR-amplified nuclear DNA sequences and via microsatellites. Diploid

54 nuclear genes were typically amplified via PCR and sequenced directly (i.e., without cloning

55 first), a practice that led to much hand-wringing over how to determine the phase of nuclear

56 haplotypes comprising the PCR product from heterozygous individuals (Palumbi and Baker

57 1994; Hare and Palumbi 1999). The phase of nuclear alleles is important because only after

58 determining phase is one able to coherently analyze alleles within populations or linked sites,

59 even for PCR products of a few hundred base pairs. Even today, particularly when PCR-

60 amplified nuclear genes are used in phylogenetics, the phase of nuclear alleles is often ignored,

61 possibly because it is not deemed important when comparing highly divergent species.

62 Recombination also had to be acknowledged and often this was accomplished by determining

63 DNA tracts within which no detectable recombination was observed. Detection of

64 recombination events was often accomplished through software focusing on phylogenetic

65 discordances among sites within a sequence or by estimating linkage disequilibrium among sites

66 within or between loci (Hudson and Kaplan 1988). Testing for and dealing with recombination,

67 for example by retaining only sections of an alignment free of detectable recombination, was and

68 continues to be important so as to ensure assumptions are not violated when building gene trees 
69 or when estimating population parameters that require either full linkage between sites within

70 loci or complete independence of sites.

71 The specific ways in which sequence data were analyzed were very much constrained by

72 the technical limits of PCR; typically data sets consisted of a few loci, each of a few hundreds

73 base pairs, and an ecosystem of software emerged around this particular format. Discordances

74 between nuclear and mitochondrial DNA came to the fore as researchers were able to directly

75 observe them in phylogenetic and phylogeographic analyses (e.g. Godinho et al. 2008), and in

76 particular in studies of hybrid zones. The effects of incomplete lineage sorting were also readily

77 visible even in analyses employing few loci. PCR of nuclear genes was very much a brute-force

78 operation, the number of loci being assayed directly proportional to the amount of effort and

79 number of PCR experiments performed. At the zenith of the (PCR) nuclear age in

80 phylogeography (Balakrishnan et al. 2010), typical studies included tens of loci, and there was

81 mounting evidence that the uncertainty of estimates of demographic parameters decreased with

82 increasing numbers of loci. In addition, population genetic theory suggested much the same: for

83 example, the optimal sampling scheme for estimating genetic diversity within single populations

84 is generally thought to maximize the number of loci at the expense of the number of individuals

85 (alleles) or length of loci (Nei and Roychoudhury 1974; Pluzhnikov and Donnelly 1996;

86 Felsenstein 2006; Carling and Brumfield 2007). Surprisingly, the optimal sampling scheme for

87 estimating genetic parameters from multiple populations partially linked by gene flow is still

88 understudied. In the PCR era, given constraints on budgets and finite ability to sample

89 individuals, there was a trade-off between the number of individuals or populations sampled and

90 the number of loci assayed. We suggest that this trade-off is partially removed with the advent

91 of next generation sequencing. 
Microsatellites and other simple sequence repeats also emerged in phylogeography in

93 1990s, following their discovery in the previous decade (Tautz and Renz 1984; Jeffreys et al.

94 1985). Although first employed extensively in the study of parentage in natural populations

95 (Burke et al. 1989; Gyllensten et al. 1990), understandably these markers swept like wildfire

96 through phylogeography. Indeed, despite the fact that microsatellites fail to capture critical

97 components of the original spirit of phylogeography - in particular phylogeography's focus on

98 phylogenetic lineages - they have historically been the most extensively used molecular marker

99 in phylogeography. Their popularity is understandable because of their hypervariability - it is

100 easy to be seduced by markers with such a large number of alleles and such potentially high

101 resolving power. On the positive side, microsatellites have provided unquestionable insight into

102 the demographic histories of literally thousands of species, and have helped expand

103 phylogeography to incorporate and synergize with sister disciplines such as population genetics,

104 landscape genetics, and even behavioral ecology. They also carry some information on the

105 relationships among alleles -- assuming a step-wise mutation model -- and in principle have the

106 ability to distinguish between recent and more ancient timescales of population divergence (e.g.

107 Fst vs. Rst comparisons). On this logic, some authors have suggested they may be useful for

108 estimating divergence times as well (Sun et al. 2009). On the down side, several authors have

109 called for a reappraisal of the utility and neutrality assumptions of microsatellites and have

110 questioned the high degree of enthusiasm for these markers in phylogeography (Brumfield et al.

111 2003; Morin et al. 2004; Zink and Barrowclough 2008; Edwards and Bensch 2009; Zink 2010;

112 Albayrak et al. 2012; Perktas et al. 2015). As put by Morin et al (2004) “...the high information

113 content [of microsatellites], a result of high mutation rates, comes at a price...”. The challenges

114 and deficiencies of microsatellites in phylogeography have been reviewed extensively elsewhere 
115 (Zink 2010), and include substantial homoplasy, making estimates of the number of mutations

116 difficult; an inability to conduct robust phylogenetic analyses, and hence offering little continuity

117 between phylogeography and phylogenetics; frequent null alleles; and difficulty comparing to

118 sequence-based markers, including mtDNA. Less well-appreciated deficiencies of

119 microsatellites include clear evidence that some simple sequence repeats are indeed functional -

120 they are often involved in gene regulation in both microbes and eukaryotic genomes - and thus

121 may be subject to selection. This last critique no doubt applies to other kinds of markers as well,

122 including SNPs, but the frequent appeal to neutrality by users of microsatellites should be

123 tempered by the increasing number of examples of functional roles for such markers (Liu et al.

124 2000; Metzgar et al. 2000; Sureshkumar et al. 2009; Tremblay et al. 2010; Grover and Sharma

125 2011; Gao et al. 2013).

126 There are no doubt still staunch defenders of microsatellites, and we do not mean to

127 suggest that SNPs, sequence-based markers, or other alternatives to microsatellites are not above

128 reproach. A major criticism of sequence based markers or SNPs in phylogeography has been the

129 paucity of such markers and their low polymorphism. While these criticisms may have been

130 valid in the PCR era, we suggest that they no longer apply meaningfully given the large number

131 of SNPs now achievable with next-generation sequencing approaches. By contrast, although the

132 number of microsatellite loci has been increasing in recent years, we do not know of efforts to

133 assay variation targeted at microsatellites using next-generation approaches. Next-generation

134 isolation of microsatellite loci, followed by PCR-based assays of variation, has been used with

135 considerable success (Abdelkrim et al. 2009; Perry and Rowe 2011; Singham et al. 2012; Curto

136 et al. 2013; Taguchi et al. 2013), but actually assaying variation and scaling up beyond PCR-

137 based assays to our knowledge has not occurred yet for microsatellites in studies of 
138 phylogeography (but see Fordyce et al. (2011) for other applications). Although it is surely too

139 early to tell, we suggest that this technical gap implies that the community does not place a high

140 priority on scaling up for microsatellites, perhaps because it is thus far comfortable with the

141 expanded power of SNPs in the next-generation sequencing era. Garrick et al. (2015) recently

142 declared that "Compared to other classes of molecular markers, DNA sequence haplotypes and

143 single nucleotide polymorphisms (SNPs) should be more informative about historical events and

144 processes ... operating over timescales most relevant to the discipline [of phylogeography]"

145 (Garrick et al. 2015). While we agree wholeheartedly with this statement, we suggest that much

146 of the community might still favor microsatellites if given the choice, particularly in comparisons

147 of closely related, endangered or very recently diverged populations. This preference, we

148 suspect, is due in part because some labs may not yet have ready access to next-generation

149 sequencing methods. But it might also be due to the perception that, due to their hypervariability,

150 microsatellites have advantages over SNPs in many contexts, especially if they can be assayed in

151 large numbers (Becquet et al. 2007; Kwong and Pemberton 2014).

152 We sought to determine whether changes in methodologies and markers used in

153 phylogeography have been driven by choice or instead more by the availability of technologies

154 adopted primarily for increasing the number of loci. We conducted a study parallel to that of

155 Garrick et al (2015) by reading abstracts for 397 papers that use microsatellites in

156 phylogeography and were published in Molecular Ecology, a major outlet for phylogeographic

157 research (Fig. 1, see legend for methods). Garrick et al. (2015)'s survey, which comprised 370

158 papers reporting on 508 single-species data sets, was interesting because it somewhat

159 unexpectedly focused on SNPs, whereas our intuition was that, among nuclear loci,

160 microsatellites were the main driver of phylogeography until recently. Our analysis confirms 
161 this suspicion: once the studies that only used mitochondrial DNA were pulled out from their

162 analysis (a total of 280 studies, comprising $73.5 \%$ of all 'SNP' studies in our sample; see Fig. 1),

163 the number of phylogeographic studies using microsats is comparable to, and sometimes exceeds,

164 that using nuclear SNPs (Fig. 1A). Intriguingly, phylogeographic studies employing only

165 mtDNA do indeed seem to be declining since 2007, at least in the pages of Molecular Ecology.

166 This decline could highlight a shift in preference within the field towards other types of genetic

167 data or a shift in preference of journals against publishing studies that rely solely on mtDNA.

168 Additionally, the year 2013 suggests a shift as studies employing nuclear SNPs begin to exceed

169 those using microsatellites. This uptick does not seem to be driven entirely by next-generation

170 sequencing, which only comprised 8 studies in our sample, suggesting that SNPs may have risen

171 in popularity independently of novel technologies and perhaps due to conceptual advances or

172 available software. Examination of microsatellite studies in all years of our sample (Fig. 1B)

173 suggest that, at best, this technology has leveled out in its popularity, particularly given the

174 increasing number of pages in the journal over time. It will be interesting to see what the next

175 five years brings in terms of the relative use of these various marker types in phylogeogeography.

176 Given the pre-eminence of Molecular Ecology in the field of phylogeography, we suggest that

177 the trends observed here may well reveal trends that will follow in time with the rest of the field.

\section{Next-generation sequencing and the rise of sequence-based markers in phylogeography}

179 Phylogeographers have appreciated for years that, despite their lower polymorphism,

180 SNPs are much more common in the genome than microsatellites (Brumfield et al. 2003). Yet

181 this point was almost moot because it was difficult if not impossible to take advantage of SNPs

182 on a scale that would capitalize on their ubiquity. The advent of next-generation sequencing will

183 likely increase the swing of the phylogeographic pendulum back in favor of SNPs and sequence- 
184 based markers once and for all. The adoption of partial genome survey methods such as Rad-seq

185 will not only yield SNPs in sufficient numbers for phylogeography, but will prescribe the use of

186 SNPs even more so than will whole-genome resequencing. Whole-genome phylogeographic

187 studies are already the norm for model species such as humans (Consortium et al. 2010; Reich et

188 al. 2010; Hammer et al. 2011; Li and Durbin 2011; Stoneking and Krause 2011) and Drosophila

189 (Yukilevich et al. 2010; Campo et al. 2013; Duchen et al. 2013; Reinhardt et al. 2014) and

190 researchers will have many options for marker types once this phase is achieved. Until that time,

191 by shifting the focus of phylogeography to sequence-based markers and SNPs, next-generation

192 sequencing methods promise to stabilize and unify phylogeographic studies in many productive

193 ways. To us they are a positive trend for phylogeography because of the many reasons that SNPs

194 have previously been considered beneficial: they provide more natural comparisons to variation

195 in organelle genomes and between studies, and, despite the challenges of recombination within

196 loci, provide natural bridges to phylogenetic analysis.

Types and consequences of next-generation sequencing approaches in phylogeography:

198 Aside from the use of next-generation sequencing approaches for isolating microsatellite loci,

199 next-generation sequencing is making inroads into phylogeography in two main ways: through

200 Rad-seq, which generates short ( 100 bp) markers, typically with one or a few SNPs per locus

201 (see Puritz et al. 2014 for a review of different Rad-seq methods); and through targeted capture

202 approaches, which can be used to target already-defined sets of loci, such as exons or

203 ultraconserved elements (UCEs) and their polymorphic flanking regions (Faircloth et al. 2012;

204 Smith et al. 2014). Although transcriptome and amplicon sequencing have also both proven

205 useful in phylogeography (Hedin et al. 2012; O'Neill et al. 2013), transcriptome sequencing will

206 likely have less direct use in purely phylogeographic investigations (as opposed to the discovery 
207 of loci under selection; see below) because of its focus on loci that are relatively conserved but

208 more likely under selection, and we predict that amplicon sequencing will ultimately prove less

209 attractive to phylogeographers because of the labor involved and the smaller number of loci that

210 can be assayed (but see Mccormack and Faircloth 2013).

211 The emerging 'core' approaches of targeted enrichment and Rad-seq promise to re-orient

212 phylogeography towards sequence-based markers in different ways because of the types of data

213 they each produce (Lemmon and Lemmon 2012; McCormack et al. 2012; McCormack et al.

214 2013). Targeted enrichment approaches yield data that can be assembled into individual

215 sequence-based markers spanning hundreds to potentially thousands of base pairs, resulting in

216 haplotypes or consensus sequences within which there may be several to many SNPs that can in

217 principle be subjected to phylogenetic analysis (Lemmon and Lemmon 2013). By contrast, Rad-

218 seq typically yields loci that are too short to analyze using traditional phylogenetic methods;

219 instead, researchers typically extract single or multiple SNPs from such Rad-loci and then

220 analyze them as individual SNPs. In many ways the two approaches provide contrasting bridges

221 to phylogenetics and classical phylogeography, as well as pointing to complementary analytical

222 approaches in the future. For example, because the loci yielded by targeted enrichment

223 approaches to phylogeography can often be analyzed using standard phylogenetic methods for

224 estimating gene trees, they provide a natural bridge to classical phylogeography. By contrast,

225 although the SNPs generated by Rad-seq can be used to estimate phylogenetic relationships of

226 populations or species ('species trees'), and indeed have been subjected to concatenation

227 approaches in early examples (Emerson et al. 2010; Merz et al. 2013), currently these markers

228 are used to bypass classical gene trees and instead estimate the species tree directly (Bryant et al.

229 2012; Rheindt et al. 2014; Chifman and Kubatko 2014). These two approaches can sometimes 
230 require different sets of analyses, and it may be that the toolkit for linked SNPs such as produced

231 by targeted enrichment is still deeper than that available for analyzing SNPs.

Although both core methods will align phylogeography squarely on the use of SNPs,

233 whether linked or unlinked in individual loci, these differences in continuity with classical 'gene

234 tree' phylogeography and analytical approaches are significant. Gene trees may be the lynchpin

235 in this phylogeographic transition. Many have suggested that, despite their centrality to the

236 origins of phylogeography (Avise et al. 1987), ultimately, gene trees are a nuisance parameter in

237 phylogeography and, if anything, can be a distraction from the key levels of analysis and primary

238 interests, which are populations and species, not genes. In this sense, Rad-seq may have the

239 practical advantage of finally freeing the community conceptually from gene trees and haplotype

240 networks, which are still a ubiquitous component of phylogeography. The ability and tendency

241 to make and interpret gene trees has resulted in heated controversies in phylogeography, such as

242 the conflicts between model-based approaches in phylogeography and more literal interpretations

243 of gene trees, such promoted by nested-clade analysis (Nielsen and Beaumont 2009; Beaumont

244 et al. 2010; Templeton 2010). It will be interesting to see how the analytical methods afforded

245 by Rad-seq versus targeted enrichment influence the next ten years of phylogeography. It may

246 be that the sheer number of loci generated by both methods moves the field forward in adopting

247 the model-based approaches that are clearly appropriate for such data sets.

$248 \quad$ Power of next generation genome subsampling methods for phylogeography: Genome

249 subsampling methods such as Rad-seq and targeted capture re-sequencing, including UCE

250 analysis, hold enormous promise for phylogeographic investigations of neutral processes such as

251 population structure, species delimitation and historical demography. Phylogeography has

252 traditionally emphasized sampling of individuals and populations over loci (Brito and Edwards 
253 2009; Garrick et al. 2015). This bias is a natural and understandable outgrowth of one of the

254 main motivations for phylogeography - to discover novel lineages of organismal biodiversity

255 within species. However, it is now better appreciated that sampling robustly for loci as well as

256 individuals is essential for increased precision of parameter estimates in phylogeography and for

257 better accounting for stochastic variation among loci (Beerli and Felsenstein 1999; Edwards and

258 Beerli 2000; Felsenstein 2001; Jennings and Edwards 2005; Carling and Brumfield 2007). The

259 sheer number of loci revealed by methods such as Rad-seq, UCE analysis or targeted capture

260 methods therefore comes as a welcomed boon over the relative dearth of loci captured in a

261 typical PCR-based study. Considering one of the core goals of phylogeography is to describe the

262 history of populations using genomic data, it should not be surprising that even a dozen loci

263 (which is typical for the heyday of PCR-based phylogeography) are unlikely to capture the

264 diverse signals of history across all chromosomes in a typical genome. In the case of Rad-seq,

265 there is a serious issue involving the bias of the technique against older haplotypes that have

266 experienced mutations in the restriction sites used to isolate DNA fragments - a bias that can

267 compromise estimates of genetic variation (Arnold et al. 2013). Furthermore, the process of

268 assembling a library from non-model species often involves grouping sequence reads by some

269 similarity threshold. The choice of these thresholds is not necessarily a straightforward process,

270 and highly divergent alleles may be inadvertently omitted prior to downstream analyses either

271 because their differences exceed preset similarity thresholds, or they increase the proportion of

272 missing data in the genotype by individual matrix (Harvey et al. 2015a; Huang and Knowles, in

273 press). Still, for other next-gen subsampling approaches, the variation revealed by next-

274 generation approaches - whether amplicon sequencing of $\sim 100$ loci or the tens of thousands of 
275 SNPs that a typical Rad-seq study reveals - is likely more than adequate for understanding the

276 basic population history of most species.

Harvey et al. (2015b) recently compared the resolving power for phylogeography of data

278 sets varying in size in terms of number and length of sequence-based markers to estimate

279 demographic parameters (effective population size, divergence time, migration rate) and species

280 history in a Neotropical songbird with deep phylogeographic breaks. They found that increasing

281 the number of loci up to 5000 provided increased resolution of the particular demographic

282 histories that they studied, but that increasing the number of loci beyond this yielded minimal

283 gains. Additionally, they found that increasing locus length past $500 \mathrm{bp}$ did not yield additional

284 improvements in resolution for the focal parameters of their study. This study therefore

285 suggested that the numbers of loci revealed by genome subsampling methods such as Rad-seq

286 are likely to be adequate for resolving population history on a variety of scales. Indeed, the

287 initial round of empirical studies using data sets produced by Rad-seq or sequence capture,

288 usually on the order of 2000-30,000 SNPs or aligned markers appear quite satisfying in so far as

289 they have revealed undiscovered phylogeographic lineages that significantly improve our

290 understanding of the fit of genomic variation to environmental and topographic barriers to gene

291 flow (e.g., Alcaide et al. 2014; Harvey and Brumfield 2015). We concur with Harvey et al.

292 (2015b) that genome subsampling methods are likely to finally provide the appropriate level of

293 genomic detail for the foreseeable future of phylogeography.

294 Natural selection and the expanding domain of phylogeography enabled by next-generation 295 sequencing

296 Classically, phylogeography has focused on the neutral demographic history of species, a 
297 goal that has been facilitated by studies on organelle genomes as well as multilocus analyses of

298 nuclear genes. However, the ability to scan genomes for thousands of loci at a time has helped

299 expand the purview of phylogeography beyond neutral demographic histories to include the

300 discovery of loci under selection. As we have seen, with the advent of diverse types of nuclear

301 markers including SNPs, phylogeography has relaxed its original focus on gene trees; purists

302 may even argue that the original definition of phylogeography included a substantial focus on

303 mitochondrial gene trees, and that use of nuclear markers with their frequent recombination

304 might constitute an expansion of the original definition of phylogeography (Avise et al. 1987).

305 In the same way, the ability to examine variation on a large scale and to focus on, for example,

306 variation in transcriptomes and exomes (e.g., Marra et al. 2014) where natural selection is likely

307 to be more prevalent, has allowed phylogeography to embrace topics such as natural selection.

308 This shift, although fascinating in its own right, was arguably not a part of Avise's original

309 vision for the field (Avise et al. 1987). However, the ability to examine large numbers of loci,

310 and to estimate robust distributions of alleles and allele frequencies across geography and the

311 genome, immediately raises the possibility of phylogeography embracing studies of natural

312 selection. Indeed, some of the most integrative studies in phylogeography thus far are those that

313 combine robust geographic sampling and historical demographic inference with investigations of

314 natural selection (Deagle et al. 2012; Jones et al. 2012a; Jones et al. 2012b; Pearse et al. 2014;

315 Schielzeth and Husby 2014; Wallberg et al. 2014). Have phylogeography and population

316 genetics become synonymous? We suggest not. If there is any attribute that distinguishes

317 phylogeography from population genetics it is robust geographic sampling of populations within

318 a species; such sampling is arguably a hallmark of phylogeography, yet is often not required, or

319 achieved, in even high quality studies of population genetics. 
321 population genetics to discover loci with a history of selection, beginning with Lewontin and

322 Krakauer's (1973) observation that $F_{\text {st }}$ outliers could be useful in identifying loci under selection.

323 The use of $F_{\text {st }}$ outliers has become common now, and, although there are caveats to the

324 interpretation of high $F_{\mathrm{st}}$ as an unambiguous signal of selection (Turner and Hahn 2010;

325 Cruickshank and Hahn 2014), the ability now to study distributions of loci makes it a useful tool,

326 especially when implemented with care and a consideration of the underlying demographic

327 history (Johnston et al. 2014; Lotterhos and Whitlock 2014). Recent studies suggest that, in fact,

328 avoiding natural selection entirely, even in the nuclear genome, may not be possible, whether

329 studying whole-genome or transcriptome variation, because the imprint of selection may be

330 much greater across the genome than originally envisioned by phylogeographers. For example,

331 the overall level of diversity in the nuclear genome displayed in a species is often taken by

332 phylogeographers to be a neutral indication of the historical effective population size,

333 summarized for single populations by the equation $\pi=4 \mathrm{~N} \mu$. However, the small range of

334 nuclear genetic diversities across species - usually considered to fall in a range of two orders of

335 magnitude and often called "Lewontin's (1974) paradox" - has been a major challenge for

336 population geneticists and has profound implications for phylogeography as well. The paradox

337 was a major impetus for the development of the nearly neutral theory, which placed emphasis on

338 interactions between selection and drift and seemed to fit available data better than the strictly

339 neutral theory (Ohta 1992; Ohta and Gillespie 1996). By postulating a nearly neutral zone in

340 which the absolute value of the product Ns was substantially less than 1, the nearly neutral

341 theory - clearly a key departure from Kimura's strictly neutral theory (Kimura 1968; Kimura

342 1983) - was able to account in part for this paradox. Although there have been many estimates 
343 of the distribution of selection coefficients for key model species (Keightley and Eyre-Walker

344 2010), until recently there were few compelling data to really test these ideas across a wide range

345 of species.

346 Two recent comparative studies of population genomics are relevant to Lewontin's

347 paradox and have important implications for phylogeography. Corbett-Detig et al. (2015)

348 conducted an exhaustive survey of genome-wide genetic variation in 40 eukaryotes and came to

349 the conclusion that the small range of genetic diversities $(\pi)$ among species could be explained in

350 part by the greater ability of natural selection to reduce genetic variation in species with large

351 population size. The implication of this paper is that for some species, particularly those with

352 large populations, selection may depress the level of neutral variation at nearly every site in the

353 genome, because selective sweeps are common and drift is relatively weak. This study has

354 profound implications for phylogeographic studies of widespread species; remarkably, the

355 frequent signals of natural selection that phylogeography has come to expect for mtDNA may

356 also apply to the nuclear genome, particularly as it applies to overall diversity in species with

357 large populations.

358 In another related study, Romiguier et al (2014) recently surveyed population variation in

359 transcriptomes of a variety of species across the tree of life. Like the study by Corbett-Detig et al.

360 (2015) this study, although comprehensive, cannot be considered phylogeography because the

361 main focus was not population history but the overall amount of variation. Whereas in some

362 cases multiple populations per species were sampled and much of the genetic variation within

363 each species may have been captured, in neither study was the general geographic sampling

364 robust enough to be considered phylogeography. Romiguier et al. (2014) came to the startling

365 conclusion that the amount of variation $(\pi)$ in the transcriptome was best predicted by life history 
366 attributes and longevity, rather than by geographic range or other aspects of strictly neutral

367 demography. Surprisingly their reasoning was largely based on a neutral argument: long-lived

368 and other species with K-selected life history traits tend to be able to sustain smaller populations,

369 and hence lower genetic diversities, than species with r-selected life history traits, because they

370 live in more stable environments with fewer long-term perturbations. By contrast, $r$-selected

371 species, which tend to have greater genetic diversity, possess the large populations that allow

372 them to colonize and persist in unstable habitats. Although ecologists will likely find merit in this

373 hypothesis, because it conceives of ecology and life history as the causal driving force behind

374 population genetic variation, it is not very satisfying from a population genomics perspective. It

375 is surprising that even negative selection on deleterious variation, traditionally considered a

376 major force in regulating intraspecific genetic variation, especially in protein-coding regions,

377 was only briefly mentioned as potentially important for explaining the positive correlation

378 between life history and the ratio of nonsynonymous to synonymous nucleotide substitutions

$379\left(d_{n} / d_{s}\right)$ within species. In this case, the smaller populations of long-lived $\mathrm{K}$ species result in

380 higher $\mathrm{d}_{\mathrm{n}} / \mathrm{d}_{\mathrm{s}}\left(\right.$ driven largely by higher $\left.\mathrm{d}_{\mathrm{n}}\right)$ due to increased fixation of deleterious mutations in

381 small populations, as envisioned by the nearly neutral theory (see also Weber et al. 2014).

382 However, Romiguier et al (2014) suggested that overall levels of synonymous substitution were

383 largely driven by effective population size, which in turn was seen as a neutral consequence of

384 life history variation. It will be important to verify the hypothesis of this work through genome-

385 wide measurements of diversity as a part of detailed phylogeograhic analyses of diverse species.

386 Selection, recombination and hitchhiking in phylogeography: The above two studies

387 provide an important contrast in how phylogeographers are beginning to think about genome-

388 wide data. In particular, the issue of linkage disequilibrium (LD) and the potential for genome- 
389 wide hitchhiking has emerged as a key factor influencing patterns of variation in the era of

390 whole-genome phylogeography. Aside from a few key emerging models for ecology and

391 evolution, such as sticklebacks, honeybees and other groups (Jones et al. 2012a; Wallberg et al.

392 2014), the phylogeography of non-model species thus far has not dealt substantially with the

393 effects of linkage on genomic variation across geographically sampled sets of populations. In

394 the PCR era, levels of LD were occasionally measured within or between the loci that could be

395 assayed, often to confirm the independence of loci, but in general the data available to

396 phylogeographers was not comprehensive enough to provide meaningful insight into the effects

397 of hitchhiking on genome-wide variation. Levels of LD can be influenced by many factors,

398 including neutral processes such as genetic drift and population bottlenecks; selective processes

399 like selective sweeps and balancing selection; and genetic processes like rates of recombination

400 and mutation (Slatkin 2008). The population recombination rate $(\rho=4 \mathrm{Nc})$ has been measured in

401 relatively few non-model species, whereas there are numerous estimates for populations of

402 humans, mice or Drosophila (Smukowski and Noor 2011).

403 Population geneticists have been measuring LD for decades, and one can calculate the

404 LD or $\mathrm{r}^{2}$ value between any pair of markers, regardless of how densely the genome is sampled or

405 whether the markers are on the same or different chromosomes. In the pre-genomic era, when

406 LD values were calculated between loci in studies that only sparsely sampled the genome, the

407 motivation was often to study the action of natural selection. But this was a very hit or miss

408 endeavor: if the candidate genes between which LD was calculated were not involved in

409 selective processes, the result was often underwhelming. By contrast, in the era of genomics,

410 when the genome can be sampled much more densely, in principle one need not know the

411 candidate genes under selection: LD can be exploited to discover loci that are the actual targets 
412 of selection without a priori suspicion that the loci measured are under selection themselves

413 (Slatkin 2008). Correlated patterns variation across the genome due to hitchhiking have been

414 used increasingly to produce a set of candidate genes responding to selection, without those

415 candidates having been assayed directly. The candidate genes are usually usually physically

416 close to or in the same linkage blocks as, the markers directly measured, and the assumption is

417 that hitchhiking on the actual targets is causing departures from neutrality, such as high $F_{s t}$, in the

418 assayed SNPs. This protocol, variably called selection mapping or association mapping, has

419 resulted in the generation of hundreds of candidate genes in emerging model species that may be

420 responding to environmental or other selective pressures (Hohenlohe et al. 2012). Increasingly

421 studies are also taking advantage of the high LD created when two species or populations

422 hybridize: chromosomal blocks deriving from each parental population can be used to identify

423 genomic regions that underlie phenotypic traits in those populations in hybrids. This method,

424 often called admixture mapping, has been used in humans extensively and increasingly in non-

425 model species (Slate and Pemberton 2007; Pallares et al. 2014)

426 However, at the dawn of the whole genome era, such protocols can be challenging to

427 implement, and can get stuck between a rock and a hard place (Fig. 2). On the one hand,

428 methods such as Rad-seq, although delivering a large number of SNPs, still sample the genome

429 sparsely, and can fall short of this goal, particularly in species where the population

430 recombination rate is high. This failure to identify actual targets of selection through selection

431 mapping arises because the genome-wide levels of $\mathrm{LD}$ can be so low as to cause the actual

432 targets of selection to be effectively unlinked (in low or average LD with) the nearest neutral site

433 whose variation is interrogated. The result will be little evidence for selection among those

434 genomic SNPs that are assayed, and many targets of selection will be missed. Clades such as 
435 Drosophila, or many bird species, likely fall into this category, and may require whole-genome

436 resequencing to more confidently identify targets of selection through hitchhiking (Backstrom et

437 al. 2006; Ellegren et al. 2012, see Fig. 3; Backstrom et al. 2013). The advantage to such species

438 with high recombination rates is that, when an outlier locus is detected, one can be sure that one

439 is relatively close to the actual target of selection, although even whole genome resequencing

440 studies in Drosophila have sometimes yielded mixed results, especially if selection is weak, very

441 recent, or acting on standing variation. On the other hand, levels of genome-wide LD in a given

442 species may be substantial because of recent population history, a history of domestication or an

443 overall low population recombination rate. Canids are good examples of this pattern (see Fig. 3

444 and Boyko et al. 2010; Boyko 2011). In such cases, even sparse sampling of the genome will

445 often uncover sites that appear to be under selection, having hitchhiked with the actual targets

446 that could be megabases away. When LD is high, larger regions of the chromosome are dragged

447 along by hitchhiking than when LD is small, and these large regions can sometimes capture

448 hundreds of genes. But in this situation, the list of candidate genes will be so long that it becomes

449 less than useful. Threespine sticklebacks (Gasterosteus aculeatus) may fall in this category:

450 Rad-seq studies routinely identify $F_{\mathrm{st}}$ outliers but the list of genes within linkage blocks can be

451 long and often provide only a vague idea of actual targets of selection (Hohenlohe et al. 2012).

452 The timing and strength of the selection event can also be important for regulating the size of the

453 hitchhiking chromosomal segments. Chromosome inversions will also protect blocks of the

454 genome from recombination, causing hundreds of genes to remain in high LD and making

455 identification of the actual targets of selection challenging or impossible without further methods

456 development. Although genome subsampling methods such as Rad-seq will in general provide a 
457 coarser picture of hitchhiking loci, even whole genome re-sequencing will not be able to

458 unambiguously identify the actual targets of selection if LD is high.

\section{Examples: Rad-seq meets selection mapping in natural populations}

460 Our thinking on the efficacy of Rad-seq to search for loci under selection issues has been

461 influenced by recent results from our laboratory. We now use two case studies, from a lizard and

462 a songbird, to illustrate the challenges of detecting selection and of homing in on the targets of

463 selection with genome subsampling methods.

$464 \quad$ Adaptation and the evolution of cold tolerance in Green Anole lizards: The green anole

465 lizard, Anolis carolinensis, is an ideal species to explore the molecular basis of climate-mediated

466 local adaptation. The only anole native to the continental United States, this species occupies the

467 highest latitudes of any of the nearly 400 species of the genus. The northern edge of the species'

468 distribution is likely limited by winter temperatures (Williams 1969), but populations do not

469 hibernate, as is common for most mid- and high- latitude reptiles. By retreating to sheltered sites

470 and basking during sun exposure, northern populations are able to remain active and periodically

471 feed in the winter months (Bishop and Echternacht 2004), despite regular ambient temperatures

472 below freezing. Additionally, populations from different climates display significant differences

473 in cold tolerance (Wilson and Echternacht 1987). The recent publication of the genome of this

474 species (Alfoldi et al. 2011) provides a unique resource for understanding the molecular

475 processes of evolutionary response to local environment and for identifying genes that may play

476 a key role in physiological divergence between populations of a non-model species. Taking

477 advantage of this opportunity, we used double digest RAD sequencing (ddRad-seq; Peterson et al. 
478 2012) to identify regions of the A. carolinensis genome associated with cold variation across the 479 species' range.

$480 \quad$ Using SphI and EcoRI restriction enzymes, we digested genomic DNA from 28

481 individuals representing 6 populations spanning the latitudinal extent of the natural range of $A$.

482 carolinensis. We genotyped 20,282 SNPs with a coverage of $\geq 10 \mathrm{X}$ for these individuals using

483 the Stacks software package (Catchen et al. 2011; Catchen et al. 2013). To search for regions of

484 the genome associated with temperature variation across the species' range, we used

485 georeferenced locality data for each population to retrieve estimates of the mean temperature of

486 the coldest quarter of the year (BIO11) from the Worldclim database (Hijmans et al. 2005). We

487 then used allele counts from the RADseq dataset to calculate Bayes factor associations and

488 Pearson correlations using the Bayenv2 software package (Gunther and Coop 2013). Variant

489 sites in the top $1 \%$ of both Bayes factor associations and Pearson correlations were retained as

490 candidate markers identifying regions of the genome that may be important for local adaptation

491 to cold (Fig. 4). This analysis resulted in 72 candidate SNPs, all of which were noncoding: 67\%

492 are located in intergenic regions, whereas $33 \%$ map to introns.

493 Several genes in this dataset may be of interest for further study due to their close

494 proximity to SNPs exhibiting geographic correlations with temperature and their potential

495 involvement in oxygen regulation, which has been proposed as a major constraint for ectotherms

496 under extreme temperature challenge (Portner et al. 2006; Portner et al. 2007). One of these

497 variants is located $40.8 \mathrm{~kb}$ upstream from the first exon of Rho-associated protein kinase 2

498 (ROCK 2), whose signaling is important for regulation of pulmonary vasculature (Riento and

499 Ridley 2003; Noma et al. 2006; Seasholtz et al. 2006; Rankinen et al. 2008). Another SNP lies

$500 \quad 1.46 \mathrm{~kb}$ upstream of the first exon of transcription factor 4 (TCF4), which is involved in 
501 regulation of breathing patterns (Zweier et al. 2007). Functional genomics studies are needed to

502 better understand the potential role and importance of these and other physiological processes to

503 temperature-mediated local adaptation within the green anole.

504 Temporal evolution of House Finch populations before and after an epizootic: The

505 House Finch (Haemorhous mexicanus), one of the most common birds in both urban and rural

506 environments in North America, is rapidly becoming a model system for avian study. It has been

507 important in studies of rapid morphological adaptation, sexual selection, evolution of disease

508 resistance, and invasion (Badyaev et al. 2012). The uniqueness of the House Finch in studies of

509 disease ecology is the result of its relationship with the pathogen Mycoplasma gallisepticum

510 (MG). This poultry-associated bacterium was first documented in the House Finch in 1994 in the

511 Washington DC area (Ley et al. 1996; Hochachka and Dhondt 2000a). MG infects the

512 respiratory tract and causes severe conjunctivitis (Hochachka and Dhondt 2000b), suppresses

513 pathogen-specific components of the immune system (Bonneaud et al. 2011), and stimulates

514 inflammatory responses (Gaunson et al. 2006; Mohammed et al. 2007; Adelman et al. 2013). The

515 pathogen spread through the eastern population rapidly, and by 1998 had caused severe declines

516 across the region, as high as $60 \%$ in some areas (Dhondt et al. 1998). Infection experiments

517 comparing gene expression responses of eastern individuals with 12 years of exposure and

518 historically unexposed individuals suggested rapid evolution of gene expression, disease

519 resistance (Bonneaud et al. 2011; Bonneaud et al. 2012) and disease tolerance (Adelman et al.

520 2013).

521 We collected a genome-wide SNP dataset using double-digest RADseq (Peterson et al.

522 2012) to identify regions of the genome with signatures of MG-mediated selection over time. As

523 in the Anolis study, we digested the genome with SphI and EcoR1, and selected fragments from 
524 276-324 base pairs long to recover homologous loci scattered randomly across the genome. In

525 this preliminary study, we sampled only 11 individuals (22 chromosomes), 5 from pre-epizootic

526 (1990) and 6 from post-epizootic (2003) populations from Alabama. We ran the Rad-seq

527 libraries on a single lane of HiSeq Illumina 2500 , generating a total of $\sim 8$ million paired-end

528 reads ( 4 million pairs), each 150 bp long. Using the Stacks pipeline (Catchen et al. 2011;

529 Catchen et al. 2013), we genotyped over 12,000 SNPs from 2223 loci (Fig. 5). Of the 7260

530 comparisons of SNPs achieving our quality threshold between these time periods, we found 167

$531(2.3 \%)$ significant shifts in allele frequency (Fisher's exact test p-value < 0.05) from 129 unique

532 loci. Of these 129 loci, $68.2 \%$ are in intergenic regions, $29.5 \%$ are in introns, 2 loci fall within

533 exons, and 1 within a 3' UTR region. Although none of these loci retain significance with

534 Bonferroni correction, we suspect that larger sample sizes of individuals from additional

535 localities will improve detection. $F_{\mathrm{st}}$ values in this collection of SNPs range from 0.208 to 1

536 (fixed differences). These regions with high $F_{\mathrm{st}}$ are in or near genes with a variety of functions.

537 One gene, PPP2R2C is involved in immune pathways in humans, and falls $13 \mathrm{~kb}$ away from a

538 SNP with an $F_{\mathrm{st}}$ of 1.

539 These two studies illustrate the promise but also the challenges of detecting selection and

540 identifying candidate genes in vertebrate genomes using a genome subsampling method such as

541 Rad-seq (Tiffin and Ross-Ibarra 2014). In both studies, most of the Rad-seq SNPs fell in

542 noncoding regions whose relationship to nearby genes was unclear. In the example from Anolis,

543 the candidate genes identified as being the closest to those SNPs that were correlated with

544 environmental variables were often quite far away from the SNP used to tag them. In the

545 example from House Finches, the number of $F_{\mathrm{st}}$ outliers in comparisons of pre- and post-

546 epizootic populations was relatively small, perhaps because the observed level of LD in House 
547 Finches is generally small, and certainly because of our small sample sizes. In the few avian

548 species that have been studied with regard to recombination rate, rates on the autosomes are

549 likely to be quite high, with levels of LD declining rapidly as one moves away from the focal

550 SNP (Backstrom et al. 2006; Bullaughey et al. 2008; Janes et al. 2009; Li and Merila 2010;

551 Ellegren 2014). We have found that LD in songbird populations, such as Red-winged Blackbirds

552 (Agelaius phoeniceus) and House Finches, falls off rapidly after a few hundred base pairs, a

553 situation very reminiscent of populations of Drosophila (Edwards and Dillon 2004; Backstrom et

554 al. 2013). In such species, LD often declines between SNPs less than 500 bp apart, which means

555 that any SNP found to exhibit high differentiation or signatures of natural selection is unlikely to

556 be useful in identifying candidate genes for a phenotypic trait even a few kb away. Thus it is

557 unclear whether recent proposals to map QTL in natural populations using SNP-chips containing

558 on the order of 10,000 SNPs will be effective (Hagen et al. 2013).

559 Recent statistical models promise new power to estimate the distribution of effect sizes of

560 loci underlying a phenotypic trait along whole chromosomes in natural populations. For

561 example, using approximately 10,000 SNPs, Santure et al. (2013) found that for most

562 chromosomes in the genome of Great Tits studied at Whyndham Woods UK, a model in which

563 the effect of a given chromosome on continuous traits like wing length and clutch size was

564 proportional to the length of that chromosome, and hence the number of genes on that

565 chromosome. By extension, this result suggests that nearly every gene has a similar - and

566 infinitesimally small - effect on variation in the focal trait. But it is also unclear whether the

567 failure to reject such a null hypothesis is also due to the relatively meager sampling of the

568 genome. Although 10,000 SNPs seems like a large number, it is relatively small in terms of 
569 capturing variation in genomic blocks in high LD, especially for vertebrate genomes on the order

570 of 1-3 Gb and in species with high population recombination rates (Edwards 2013).

571 Measuring hitchhiking is crucial enough to the expanding domain of phylogeography that

572 we predict that, ultimately, the field will forsake genome subsampling approaches for

573 phylogeographically informed whole-genome resequencing studies. We are beginning to see the

574 first glimpses of such studies in genomically unstudied species (e.g., Ojeda et al. 2014), and the

575 results are as exciting as they are informative about the determinants of genomic variation and

576 structure.

$577 \quad$ From phylogeography to genotype to phenotype: Loci whose variation has been

578 influenced by natural selection are often also loci that underlie phenotypic traits. The search for

579 loci underlying natural variation in phenotypic traits is a major thrust of modern evolutionary

580 biology (Hoekstra et al. 2006; Hoekstra and Coyne 2007; Ellegren and Sheldon 2008; Rebeiz et

581 al. 2009; Hiller et al. 2012; Jones et al. 2012b). Of the many methods for identifying such loci -

582 including QTL and linkage mapping using pedigrees and crosses, or "systems genetics", in

583 which multiple kinds of genomic, transcriptomic, and metabolomic data are integrated together

584 (Feltus 2014) - association mapping is probably the method most closely allied to

585 phylogeography. This alliance arises because association mapping uses population comparisons

586 to find genomic loci that correlate with the distribution of a particular phenotype (Stinchcombe

587 and Hoekstra 2008; reviewed in Kratochwil and Meyer 2015). Others have made a similar

588 connection between landscape genetics and the search for loci underlying adaptive phenotypes

589 (Jones et al. 2013). Association mapping has significant promise for closing the genotype-

590 phenotype gap in non-model species and in many contexts has more statistical power than does

591 mapping with pedigrees or controlled crosses (Schielzeth and Husby 2014). Indeed, the 
592 emerging trend is toward simultaneous inference of population history and identification of loci

593 associated with phenotypic traits (e.g., Fumigalli et al. 2011; Linnen et al. 2013). Association

594 mapping is likely to be most powerful in situations where most of the genomic variation is

595 shared among a group of closely related populations or species, perhaps connected by high gene

596 flow, but there exist marked phenotypic differences among those populations (Axelsson et al.

597 2013; Cullingham et al. 2014; Schielzeth and Husby 2014). In such situations, association

598 mapping should reveal similar allele frequencies across nearly the entire genome, often due to

599 shared standing variation, in both control and comparison populations, yet these populations

600 should differ at loci whose variation correlates with the divergent phenotypes of interest.

601 Precisely this situation has been found in those emerging cases where association mapping or

602 candidate gene investigation has proved useful in non-model species, including several plants

603 (Comeault et al. 2014; Cullingham et al. 2014; Johnston et al. 2014; Pearse et al. 2014; Roesti et

604 al. 2014). Use of candidate genes in such situations can also be highly informative (Uy et al.

605 2009). Indeed, when populations have experienced a moderate history of divergence, such that

606 the compared genomes differ at many sites due to neutral demographic divergence, it becomes

607 essential to correct for such substructuring so as not to spuriously implicate loci showing strong

608 allele frequency differences in the origin of phenotypic traits that between those populations

609 (Pritchard et al. 2000; Patterson et al. 2006; Price et al. 2006; Price et al. 2010).

\section{Conclusions}

611 This is an exciting time for phylogeography. We have a growing number of examples of

612 studies in which employment of next-generation sequencing methods has yielded high resolution

613 substructuring within species at a level of detail that far exceeds that formerly yielded by single

614 locus mtDNA or microsatellite studies. It is now clear that a few thousand loci such as is 
615 typically yielded by methods such as Rad-seq may well be adequate to discover the major

616 phylogeographic lineages within a species. Although the finer details of the history of species

617 can always be clarified further with increasing genomic sampling (or individual sampling), many

618 of these details are likely lost to historical reconstruction because of their age or their mild

619 imprint on the pattern of genome-wide variation. While we expect whole-genome resequencing

620 to become more common in phylogeography, it is unclear whether this method will be overkill if

621 the focus is strictly on the core purview of phylogeography, namely reconstruction of the

622 phylogenetic lineages and neutral demography within species. What is clear is that next-

623 generation methods are causing a resurgence in the use of SNPs as opposed to microsatellites,

624 enabling easier comparisons among loci and species and providing a uniform framework for

625 comparative phylogeography (Hickerson et al. 2010; Andrew et al. 2013).

626 But next-generation sequencing has also broken down the conceptual edges of

627 phylogeography, resulting in an expanded purview that has blurred the lines between core

628 phylogeographic foci and other areas of related interest, such as identifying loci with a history of

629 natural selection and underlying variation in adaptive traits. Studies combining historical

630 reconstruction of phylogeographic history and a search for such adaptive loci are becoming more

631 common (Deagle et al. 2012; Jones et al. 2012a; Jones et al. 2012b; Pearse et al. 2014; Wallberg

632 et al. 2014), and the goal of identifying loci underlying adaptive traits is often as important as

633 understanding the demographic history of a set of populations. This conceptually expanded

634 phylogeography marks an important phase in the evolution of the field, and although always a

635 glimmer in the eye of phylogeographers, has arguably been driven due to the recent arrival of

636 high-throughput genomic approaches. As phylogeography expands its purview, it is becoming

637 clear that genome subsampling methods such as Rad-seq, while extremely powerful for 
638 identifying phylogeographic clusters within species, may be inadequate for identifying loci that

639 are targets of natural selection or linked to the true targets. Whole-genome resequencing will

640 likely emerge as the standard tool, if not for traditional phylogeographic investigations, then

641 certainly in the quest for loci underlying quantitative traits in natural populations and their

642 history of divergence within species.

643

644 Acknowledgements: We thank Utku Perktas for inviting us to contribute to this volume.

645 We thank Tim Sackton, Ryan Garrick and Bryan Carstens for helpful discussion and sharing of

646 data, and two anonymous reviewers and Ryan Garrick for helpful comments on the manuscript.

647 


\section{References}

650

651

652

653

654

655

656

657

658

659

660

661

662

663

664

665

666

667

668

669

670

671

672

673

674

675

676

677

678

679

680

681

682

683

684

685

686

687

688

689

690

691

Abdelkrim, J., B. Robertson, J.-A. Stanton, and N. Gemmell. 2009. Fast, cost-effective development of species-specific microsatellite markers by genomic sequencing. BioTechniques 46:185-192.

Adelman, J. S., L. Kirkpatrick, J. L. Grodio, and D. M. Hawley. 2013. House Finch populations differ in early inflammatory signaling and pathogen tolerance at the peak of Mycoplasma gallisepticum infection. The American Naturalist 181:674-689.

Albayrak, T., J. Gonzalez, S. V. Drovetski, and M. Wink. 2012. Phylogeography and population structure of Kruper's Nuthatch Sitta krueperi from Turkey based on microsatellites and mitochondrial DNA. J Ornithol 153:405-411.

Alcaide, M., E. S. C. Scordato, T. D. Price, and D. E. Irwin. 2014. Genomic divergence in a ring species complex. Nature 511:83-85.

Andrew, R. L., L. Bernatchez, A. Bonin, C. A. Buerkle, B. C. Carstens, B. C. Emerson, D. Garant, T. Giraud, N. C. Kane, S. M. Rogers, J. Slate, H. Smith, V. L. Sork, G. N. Stone, T. H. Vines, L. Waits, A. Widmer, and L. H. Rieseberg. 2013. A road map for molecular ecology. Mol Ecol 22:2605-2626.

Arnold, B., R. B. Corbett-Detig, D. Hartl, and K. Bomblies. 2013. RADseq underestimates diversity and introduces genealogical biases due to nonrandom haplotype sampling. Mol Ecol 22:3179-3190.

Avise, J. C., J. Arnold, R. M. Ball, E. Bermingham, T. Lamb, J. E. Neigel, C. A. Reeb, and N. C. Saunders. 1987. Intraspecific phylogeography: the mitochondrial DNA bridge between population genetics and systematics. Annu Rev Ecol Syst 18:489-522.

Axelsson, E., A. Ratnakumar, M. L. Arendt, K. Maqbool, M. T. Webster, M. Perloski, O. Liberg, J. M. Arnemo, A. Hedhammar, and K. Lindblad-Toh. 2013. The genomic signature of dog domestication reveals adaptation to a starch-rich diet. Nature 495:360-364.

Backstrom, N., A. Qvarnstrom, L. Gustafsson, and H. Ellegren. 2006. Levels of linkage disequilibrium in a wild bird population. Biol Lett 2:435-438.

Backstrom, N., D. Shipilina, M. P. K. Blom, and S. V. Edwards. 2013. Cis-regulatory sequence variation and association with Mycoplasma load in natural populations of the house finch (Carpodacus mexicanus). Ecology and Evolution 3:655-666.

Badyaev, A. V., V. Belloni, and G. E. Hill. 2012. House Finch (Haemorhous mexicanus)in A. Poole, ed. Ithaca, Cornell Lab of Ornithology.

Balakrishnan, C. N., L. J. Y., and S. V. Edwards. 2010. Phylogeography and phylogenetics in the nuclear age, Pages 65-88 in P. Grant, and R. Grant, eds. Searching for the Causes of Evolution: From Field Observations to Mechanisms. Princeton, NJ, Princeton University Press.

Ballard, J. W. O., and N. Pichaud. 2014. Mitochondrial DNA: more than an evolutionary bystander. Funct Ecol 28:218-231.

Ballard, J. W. O., and M. C. Whitlock. 2004. The incomplete natural history of mitochondria. Mol Ecol 13:729-744.

Beaumont, M. A., R. Nielsen, C. Robert, J. Hey, O. Gaggiotti, L. Knowles, A. Estoup, M. Panchal, J. Corander, M. Hickerson, S. A. Sisson, N. Fagundes, L. Chikhi, P. Beerli, R. Vitalis, J. M. Cornuet, J. Huelsenbeck, M. Foll, Z. H. Yang, F. Rousset, D. Balding, and 

Ecol 19:436-446.

Becquet, C., N. Patterson, A. C. Stone, M. Przeworski, and D. Reich. 2007. Genetic structure of chimpanzee populations. PLoS Genet 3:e66.

Beerli, P., and J. Felsenstein. 1999. Maximum-likelihood estimation of migration rates and effective population numbers in two populations using a coalescent approach. Genetics 152:763-773.

Bonneaud, C., S. L. Balenger, A. F. Russell, J. Zhang, G. E. Hill, and S. V. Edwards. 2011. Rapid evolution of disease resistance is accompanied by functional changes in gene expression in a wild bird. Proceedings of the National Academy of Sciences 108:78667871.

Bonneaud, C., S. L. Balenger, J. Zhang, S. V. Edwards, and G. E. Hill. 2012. Innate immunity and the evolution of resistance to an emerging infectious disease in a wild bird. Mol Ecol 21:2628-2639.

Boyko, A. R. 2011. The domestic dog: man's best friend in the genomic era. Genome Biol 12:216.

Boyko, A. R., P. Quignon, L. Li, J. J. Schoenebeck, J. D. Degenhardt, K. E. Lohmueller, K. Y. Zhao, A. Brisbin, H. G. Parker, B. M. vonHoldt, M. Cargill, A. Auton, A. Reynolds, A. G. Elkahloun, M. Castelhano, D. S. Mosher, N. B. Sutter, G. S. Johnson, J. Novembre, M. J. Hubisz, A. Siepel, R. K. Wayne, C. D. Bustamante, and E. A. Ostrander. 2010. A Simple Genetic Architecture Underlies Morphological Variation in Dogs. Plos Biol 8:e10000451.

Brito, P., and S. V. Edwards. 2009. Multilocus phylogeography and phylogenetics using sequence-based markers. Genetica 135:439-455.

Brumfield, R., D. A. Nickerson, P. Beerli, and S. V. Edwards. 2003. The utility of single nucleotide polymorphisms in inferences of population history. Trends in Ecology and Evolution 18:249-256.

Bryant, D., R. Bouckaert, J. Felsenstein, N. A. Rosenberg, and A. RoyChoudhury. 2012. Inferring Species Trees Directly from Biallelic Genetic Markers: Bypassing Gene Trees in a Full Coalescent Analysis. Mol Biol Evol 29:1917-1932.

Bullaughey, K., M. Przeworski, and G. Coop. 2008. No effect of recombination on the efficacy of natural selection in primates. Genome Res 18:544-554.

Burke, T., N. B. Davies, M. W. Bruford, and B. J. Hatchwell. 1989. Parental Care and MatingBehavior of Polyandrous Dunnocks Prunella-Modularis Related to Paternity by DNA Fingerprinting. Nature 338:249-251.

Campo, D., K. Lehmann, C. Fjeldsted, T. Souaiaia, J. Kao, and S. V. Nuzhdin. 2013. Wholegenome sequencing of two North American Drosophila melanogasterpopulations reveals genetic differentiation and positive selection. Mol Ecol 22:5084-5097.

Carling, M. D., and R. T. Brumfield. 2007. Gene sampling strategies for multi-locus population estimates of genetic diversity (theta). Plos One 2:e160.

Catchen, J., P. A. Hohenlohe, S. Bassham, A. Amores, and W. A. Cresko. 2013. Stacks: an analysis tool set for population genomics. Mol Ecol 22:3124-3140.

Catchen, J. M., A. Amores, P. Hohenlohe, W. Cresko, and J. H. Postlethwait. 2011. Stacks: building and genotyping Loci de novo from short-read sequences. G3 1:171-182.

Chifman, J., and L. Kubatko. 2014. Quartet inference from SNP data under the coalescent model. Bioinformatics 30:3317-3324. 
Comeault, A. A., V. Soria-Carrasco, Z. Gompert, T. E. Farkas, C. A. Buerkle, T. L. Parchman, and P. Nosil. 2014. Genome-Wide Association Mapping of Phenotypic Traits Subject to a Range of Intensities of Natural Selection in Timema cristinae*. Am Nat 183:711-727.

\section{Consortium, G. P., G. R. Abecasis, D. Altshuler, A. Auton, L. D. Brooks, R. M. Durbin, R. A.} Gibbs, M.E. Hurles, and G. A. McVean. 2010. A map of human genome variation from population-scale sequencing. Nature 467:1061-1073.

Corbett-Detig, R. B., D. L. Hartl, and T. B. Sackton. 2015. Natural selection constrains neutral diversity across a wide range of species. Plos Biol 13:e1002112.

Cruickshank, T. E., and M. W. Hahn. 2014. Reanalysis suggests that genomic islands of speciation are due to reduced diversity, not reduced gene flow. Mol Ecol 23:3133-3157.

Cullingham, C. I., J. E. K. Cooke, and D. W. Coltman. 2014. Cross-species outlier detection reveals different evolutionary pressures between sister species. New Phytologist 204:215229.

Curto, M. A., L. R. Tembrock, P. Puppo, M. Nogueira, M. P. Simmons, and H. Meimberg. 2013. Evaluation of microsatellites of Catha edulis (qat; Celastraceae) identified using pyrosequencing. Biochem Syst Ecol 49:1-9.

Deagle, B. E., F. C. Jones, Y. G. F. Chan, D. M. Absher, D. M. Kingsley, and T. E. Reimchen. 2012. Population genomics of parallel phenotypic evolution in stickleback across streamlake ecological transitions. P R Soc B 279:1277-1286.

Dhondt, A. A., D. L. Tessaglia, and R. L. Slothower. 1998. Epidemic mycoplasmal conjunctivitis in house finches from eastern North America. J. Wildlife Dis. 34:265-280.

Duchen, P., D. Živković, S. Hutter, W. Stephan, and S. Laurent. 2013. Demographic inference reveals African and European admixture in the North American Drosophila melanogaster population. Genetics 193:291-301.

Edwards, S., and S. Bensch. 2009. Looking forwards or looking backwards in avian phylogeography? A comment on Zink and Barrowclough 2008. Mol Ecol 18:2930-2933.

Edwards, S. V. 2013. Next-generation QTL mapping: crowdsourcing SNPs, without pedigrees. Mol Ecol 22:3885-3887.

Edwards, S. V., and P. Beerli. 2000. Perspective: Gene divergence, population divergence, and the variance in coalescence time in phylogeographic studies. Evolution 54:1839-1854.

Edwards, S. V., and M. Dillon. 2004. Hitchhiking and recombination in birds: evidence from Mhc-linked and unlinked loci in red-winged blackbirds (Agelaius phoeniceus). Genet Res 84:175-192.

Ellegren, H. 2014. Genome sequencing and population genomics in non-model organisms. Trends Ecol Evol 29:51-63.

Ellegren, H., and B. C. Sheldon. 2008. Genetic basis of fitness differences in natural populations. Nature 452:169-175.

Ellegren, H., L. Smeds, R. Burri, P. I. Olason, N. Backstrom, T. Kawakami, A. Kunstner, H. Makinen, K. Nadachowska-Brzyska, A. Qvarnstrom, S. Uebbing, and J. B. W. Wolf. 2012. The genomic landscape of species divergence in Ficedula flycatchers. Nature 491:756-760.

Emerson, K. J., Merz, C. R., Catchen, J. M., Hohenlohe, P. a, Cresko, W. a, Bradshaw, W. E., \& Holzapfel, C. M. (2010). Resolving postglacial phylogeography using high-throughput sequencing. Proceedings of the National Academy of Sciences of the United States of America, 107(37), 16196-16200. doi:10.1073/pnas.1006538107 
782

783

784

785

786

787

788

789

790

791

792

793

794

795

796

797

798

799

800

801

802

803

804

805

806

807

808

809

810

811

812

813

814

815

816

817

818

819

820

821

822

823

824

825

826

827

Excoffier, L., and N. Ray. 2008. Surfing during population expansions promotes genetic revolutions and structuration. TREE 23:347-351.

Faircloth, B. C., J. E. Mccormack, N. G. Crawford, M. G. Harvey, R. T. Brumfield, and T. C. Glenn. 2012. Ultraconserved elements anchor thousands of genetic markers spanning multiple evolutionary timescales. Syst Biol 61:717-726.

Felsenstein, J. 2001. Accuracy of coalescent likelihood estimates: do we need more sites, more sequences, or more loci? Mol Biol Evol 23:691-700.

Felsenstein, J. 2006. Accuracy of coalescent likelihood estimates: do we need more sites, more sequences, or more loci? Mol Biol Evol 23:691-700.

Feltus, F. A. 2014. Systems genetics: a paradigm to improve discovery of candidate genes and mechanisms underlying complex traits. Plant science : an international journal of experimental plant biology 223:45-48.

Fordyce, S. L., M. C. Ávila-Arcos, E. Rockenbauer, C. Børsting, R. Frank-Hansen, F. T. Petersen, E. Willerslev, A. J. Hansen, N. Morling, and M. T. P. Gilbert. 2011. Highthroughput sequencing of core STR loci for forensic genetic investigations using the Roche Genome Sequencer FLX platform. BioTech. 51:127-133.

Fumagalli, M., Sironi, M., Pozzoli, U., Ferrer-Admetlla, A., Ferrer-Admettla, A., Pattini, L., \& Nielsen, R. 2011. Signatures of environmental genetic adaptation pinpoint pathogens as the main selective pressure through human evolution. PLoS Genetics, 7(11), e1002355. doi:10.1371/journal.pgen.1002355

Gao, C. H., X. D. Ren, A. S. Mason, J. N. Li, W. Wang, M. L. Xiao, and D. H. Fu. 2013. Revisiting an important component of plant genomes: microsatellites. Functional Plant Biology 40:645-661.

Garrick, R. C., I. A. S. Bonatelli, C. Hyseni, A. Morales, T. A. Pelletier, M. F. Perez, E. Rice, J. D. Satler, R. E. Symula, M. T. C. Thomé, and B. C. Carstens. 2015. The evolution of phylogeographic datasets. Mol. Ecol. 24:1164-1171.

Gaunson, J. E., C. J. Philip, K. G. Whithear, and G. F. Browning. 2006. The cellular immune response in the tracheal mucosa to Mycoplasma gallisepticum in vaccinated and unvaccinated chickens in the acute and chronic stages of disease. Vaccine 24:2627-2633.

Gerber, A. S., R. Loggins, S. Kumar, and T. E. Dowling. 2001. Does nonneutral evolution shape observed patterns of DNA variation in animal mitochondrial genomes? Annual Review of Genetics 35:539-566.

Godinho, R., E. Crespo, and N. Ferrand. 2008. The limits of mtDNA phylogeography: complex patterns of population history in a highly structured Iberian lizard are only revealed by the use of nuclear markers. Mol Ecol 17:4670-4683.

Grover, A., and P. C. Sharma. 2011. Is spatial occurrence of microsatellites in the genome a determinant of their function and dynamics contributing to genome evolution? Curr Sci India 100:859-869.

Gunther, T., and G. Coop. 2013. Robust Identification of Local Adaptation from Allele Frequencies. Genetics 195:205-220.

Gyllensten, U. B., S. Jakobsson, and H. Temrin. 1990. No Evidence for Illegitimate Young in Monogamous and Polygynous Warblers. Nature 343:168-170.

Hagen, I. J., A. M. Billing, B. Ronning, S. A. Pedersen, H. Parn, J. Slate, and H. Jensen. 2013. The easy road to genome-wide medium density SNP screening in a non-model species: development and application of a 10K SNP-chip for the house sparrow (Passer domesticus). Molecular Ecology Resources 13:429-439. 
828

829

830

831

832

833

834

835

836

837

838

839

840

841

842

843

844

845

846

847

848

849

850

851

852

853

854

855

856

857

858

859

860

861

862

863

864

865

866

867

868

869

870

871

Hammer, M., A. Woerner, F. Mendez, J. Watkins, and J. Wall. 2011. Genetic evidence for archaic admixture in Africa. Proceedings of the National Academy of Sciences 108:15123-15128.

Hare, M. P., and S. R. Palumbi. 1999. The accuracy of heterozygous base calling from diploid sequence and resolution of haplotypes using allele-specific sequencing. Mol Ecol 8:17501752.

Harvey, M. G., and R. T. Brumfield. 2015. Genomic variation in a widespread Neotropical bird (Xenops minutus) reveals divergence, population expansion, and gene flow. Mol Phylogenet Evol 83:305-316.

Harvey, M. G., C. D. Judy, G. F. Seeholzer, J. M. Maley, G. R. Graves, and R. T. Brumfield. 2015a. Similarity thresholds used in DNA sequence assembly from short reads can reduce the comparability of population histories across species. PeerJ 3:e895.

Harvey, M. G., B. T. Smith, T. C. Glenn, B. C. Faircloth, and R. T. Brumfield. 2015b. Sequence Capture Versus Restriction Site Associated DNA Sequencing for Phylogeography. arXiv:1312.6439.

Hedin, M., J. Starrett, S. Akhter, A. L. Schonhofer, and J. W. Shultz. 2012. Phylogenomic resolution of paleozoic divergences in harvestmen (Arachnida, Opiliones) via analysis of next-generation transcriptome data. Plos One 7:e42888.

Hickerson, M. J., B. C. Carstens, J. Cavender-Bares, K. A. Crandall, C. H. Graham, J. B. Johnson, L. Rissler, P. F. Victoriano, and A. D. Yoder. 2010. Phylogeography's past, present, and future: 10 years after Avise, 2000. Mol Phylogenet Evol 54:291-301.

Hijmans, R. J., S. E. Cameron, J. L. Parra, P. G. Jones, and A. Jarvis. 2005. Very high resolution interpolated climate surfaces for global land areas. International Journal of Climatology 25:1965-1978.

Hiller, M., B. T. Schaar, V. B. Indjeian, D. M. Kingsley, L. R. Hagey, and G. Bejerano. 2012. A "forward genomics" approach links genotype to phenotype using independent phenotypic losses among related species. Cell reports 2:817-823.

Hochachka, W. M., and A. A. Dhondt. 2000a. Density-dependent decline of host abundance resulting from a new infectious disease. Proc Natl Acad Sci U S A 97:5303-5306.

Hochachka, W. M., and A. A. Dhondt. 2000b. Density-dependent decline of host abundance resulting from a new infectious disease. Proceedings of the National Academy of Sciences 97:5303-5306.

Hoekstra, H. E., and J. A. Coyne. 2007. The locus of evolution: Evo devo and the genetics of adaptation. Evolution 61:995-1016.

Hoekstra, H. E., R. J. Hirschmann, R. A. Bundey, P. A. Insel, and J. P. Crossland. 2006. A single amino acid mutation contributes to adaptive beach mouse color pattern. Science 313:101104.

Hohenlohe, P. A., S. Bassham, M. Currey, and W. A. Cresko. 2012, Extensive linkage disequilibrium and parallel adaptive divergence across threespine stickleback genomes. Phil. Trans. Roy. Soc. B 367:395-408.

Huang, H., and L.L. Knowles. In press. Unforseen consequences of excluding missing data from next-generation sequences: simulation study of RAD sequences. Syst. Biol.

Hudson, R. R., and N. L. Kaplan. 1988. The coalescent process in models with selection and recombination. Genetics 120:831-840. 
872

873

874

875

876

877

878

879

880

881

882

883

884

885

886

887

888

889

890

891

892

893

894

895

896

897

898

899

900

901

902

903

904

905

906

907

908

909

910

911

912

913

914

915

916

917

Janes, D. E., T. Ezaz, J. A. M. Graves, and S. V. Edwards. 2009. Recombination and nucleotide diversity in the sex chromosomal pseudoautosomal region of the Emu, Dromaius novaehollandiae. J Hered 100:125-136.

Jeffreys, A. J., V. Wilson, and S. L. Thein. 1985. Hypervariable Minisatellite Regions in Human DNA. Nature 314:67-73.

Jennings, W. B., and S. V. Edwards. 2005. Speciational history of Australian grass finches (Poephila) inferred from 30 gene trees. Evolution 59:2033-2047.

Jobling, M. A. 2012. The impact of recent events on human genetic diversity. Philos T R Soc B 367:793-799.

Johnston, S. E., P. Orell, V. L. Pritchard, M. P. Kent, S. Lien, E. Niemela, J. Erkinaro, and C. R. Primmer. 2014. Genome-wide SNP analysis reveals a genetic basis for sea-age variation in a wild population of Atlantic salmon (Salmo salar). Mol Ecol 23:3452-3468.

Jones, F. C., Y. F. Chan, J. Schmutz, J. Grimwood, S. D. Brady, A. M. Southwick, D. M. Absher, R. M. Myers, T. E. Reimchen, B. E. Deagle, D. Schluter, and D. M. Kingsley. 2012a. A Genome-wide SNP Genotyping Array Reveals Patterns of Global and Repeated SpeciesPair Divergence in Sticklebacks. Curr Biol 22:83-90.

Jones, F. C., M. G. Grabherr, Y. F. Chan, P. Russell, E. Mauceli, J. Johnson, R. Swofford, M. Pirun, M. C. Zody, S. White, E. Birney, S. Searle, J. Schmutz, J. Grimwood, M. C. Dickson, R. M. Myers, C. T. Miller, B. R. Summers, A. K. Knecht, S. D. Brady, H. L. Zhang, A. A. Pollen, T. Howes, C. Amemiya, E. S. Lander, F. Di Palma, K. LindbladToh, D. M. Kingsley, B. I. G. S. Platf, and W. G. A. Team. 2012b. The genomic basis of adaptive evolution in threespine sticklebacks. Nature 484:55-61.

Jones, M. R., B. R. Forester, A. I. Teufel, R. V. Adams, D. N. Anstett, B. A. Goodrich, E. L. Landguth, S. Joost, and S. Manel. 2013. Integrating landscape genomics nd spatially explicit approaches to detect loci under seletion in clinal populatoins. Evolution 67:34553468.

Keightley, P. D., and A. Eyre-Walker. 2010. What can we learn about the distribution of fitness effects of new mutations from DNA sequence data? Philosophical transactions of the Royal Society of London. Series B, Biological sciences 365:1187-1193.

Kimura, M. 1968. Evolutionary rate at the molecular level. Nature 217:624-626.

Kimura, M. 1983, The Neutral Theory of Molecular Evolution. Cambridge, Cambridge University Press.

Kratochwil, C. F., and A. Meyer. 2015. Closing the genotype-phenotype gap: Emerging technologies for evolutionary genetics in ecological model vertebrate systems. Bioessays 37:213-226.

Kwong, M., and T. J. Pemberton. 2014. Sequence differences at orthologous microsatellites inflate estimates of human-chimpanzee differentiation. Bmc Genomics 15:990.

Lemmon, A. R., and E. M. Lemmon. 2012. High-Throughput Identification of Informative Nuclear Loci for Shallow-Scale Phylogenetics and Phylogeography. Syst Biol 61:745761.

Lemmon, E. M., and A. R. Lemmon. 2013. High-Throughput Genomic Data in Systematics and Phylogenetics. Annual Review of Ecology, Evolution, and Systematics 44:99-121.

Lewontin, R. 1974, The Genetic Basis of Evolutionary Change. New York, Columbia University Press.

Lewontin, R. C., and J. Krakauer. 1973. Distribution of gene frequency as a test of theory of selective neutrality of polymorphisms. Genetics 74:175-195. 
Ley, D. H., J. E. Berkhoff, and J. M. McLaren. 1996. Mycoplasma gallisepticum isolated from house finches (Carpodacus mexicanus) with conjunctivitis. Avian Diseases. 40:480-483. 
Nielsen, R., and M. A. Beaumont. 2009. Statistical inferences in phylogeography. Mol Ecol 18:1034-1047.

Noma, K., N. Oyama, and J. K. Liao. 2006. Physiological role of ROCKs in the cardiovascular system. American Journal of Physiology-Cell Physiology 290:C661-C668.

O'Neill, E. M., R. Schwartz, C. T. Bullock, J. S. Williams, H. B. Shaffer, X. Aguilar-Miguel, G. Parra-Olea, and D. W. Weisrock. 2013. Parallel tagged amplicon sequencing reveals major lineages and phylogenetic structure in the North American tiger salamander (Ambystoma tigrinum) species complex. Mol Ecol 22:111-129.

Ohta, T. 1992. The nearly neutral theory of molecular evolution. Annu Rev Ecol Syst 23:263286.

Ohta, T., and J. H. Gillespie. 1996. Development of Neutral and Nearly Neutral Theories. Theor Popul Biol 49:128-142.

Ojeda, D. I., B. Dhillon, C. K. M. Tsui, and R. C. Hamelin. 2014. Single-nucleotide polymorphism discovery in Leptographium longiclavatum, a mountain pine beetleassociated symbiotic fungus, using whole-genome resequencing. Molecular Ecology Resources 14:401-410.

Pallares, L. F., B. Harr, L. M. Turner, and D. Tautz. 2014. Use of a natural hybrid zone for genomewide association mapping of craniofacial traits in the house mouse. Mol Ecol 23:5756-5770.

Palumbi, S. R., and C. S. Baker. 1994. Contrasting Population-Structure from Nuclear Intron Sequences and Mtdna of Humpback Whales. Mol Biol Evol 11:426-435.

Patterson, N., A. L. Price, and D. Reich. 2006. Population structure and eigenanalysis. PLoS Genet Volume 2:e190.

Pearse, D. E., M. R. Miller, A. Abadia-Cardoso, and J. C. Garza. 2014. Rapid parallel evolution of standing variation in a single, complex, genomic region is associated with life history in steelhead/rainbow trout. P R Soc B 281:20140012.

Perktaş, U., Gür, H., Sağlam, İ. K., \& Quintero, E. 2015. Climate-driven range shifts and demographic events over the history of Kruper's Nuthatch Sitta krueperi. Bird Study, 62: 14-28. doi:10.1080/00063657.2014.977220

Perry, J. C., and L. Rowe. 2011. Rapid Microsatellite Development for Water Striders by NextGeneration Sequencing. J Hered 102:125-129.

Peterson, B. K., J. N. Weber, E. H. Kay, H. S. Fisher, and H. E. Hoekstra. 2012. Double Digest RADseq: An Inexpensive Method for De Novo SNP Discovery and Genotyping in Model and Non-Model Species. Plos One 7:e37135.

Pluzhnikov, A., and P. Donnelly. 1996. Optimal sequencing strategies for surveying molecular genetic diversity. Genetics 144:1247-1262.

Portner, H. O., A. F. Bennett, F. Bozinovic, A. Clarke, M. A. Lardies, M. Lucassen, B. Pelster, F. Schiemer, and J. H. Stillman. 2006. Trade-offs in thermal adaptation: The need for a molecular to ecological integration. Physiological and Biochemical Zoology 79:295-313.

Portner, H. O., L. Peck, and G. Somero. 2007. Thermal limits and adaptation in marine Antarctic ectotherms: an integrative view. Philos T R Soc B 362:2233-2258.

Price, A. L., N. J. Patterson, R. M. Plenge, M. E. Weinblatt, N. A. Shadick, and D. Reich. 2006. Principal components analysis corrects for stratification in genome-wide association studies. Nat Genet 38:904-909.

Price, A. L., N. A. Zaitlen, D. Reich, and N. Patterson. 2010. New approaches to population stratification in genome-wide association studies. Nat Rev Genet 11:459-463. 
Pritchard, J. K., M. Stephens, and P. Donnelly. 2000. Inference of population structure using multilocus genotype data. Genetics 155:945-959.

Puritz, J. B., M. V. Matz, R. J. Toonen, J. N. Weber, D. I. Bolnick, and C. E. Bird. 2014. Demystifying the RAD fad. Mol Ecol 23:5937-5942.

Rand, D.M. 2001. The units of selection on mitochondrial DNA. Annu. Rev. Ecol. Syst. 32:415448.

Rankinen, T., T. Church, T. Rice, N. Markward, S. N. Blair, and C. Bouchard. 2008. A Major Haplotype Block at the Rho-Associated Kinase 2 Locus Is Associated with a Lower Risk of Hypertension in a Recessive Manner: The HYPGENE Study. Hypertension Research 31:1651-1657.

Rebeiz, M., J. E. Pool, V. A. Kassner, C. F. Aquadro, and S. B. Carroll. 2009. Stepwise Modification of a Modular Enhancer Underlies Adaptation in a Drosophila Population. Science 326:1663-1667.

Reich, D., R. E. Green, M. Kircher, J. Krause, N. Patterson, E. Y. Durand, B. Viola, A. W. Briggs, U. Stenzel, P. L. F. Johnson, T. Maricic, J. M. Good, T. Marques-Bonet, C. Alkan, Q. Fu, S. Mallick, H. Li, M. Meyer, E. E. Eichler, M. Stoneking, M. Richards, S. Talamo, M. V. Shunkov, A. P. Derevianko, J.-J. Hublin, J. Kelso, M. Slatkin, and S. Pääbo. 2010. Genetic history of an archaic hominin group from Denisova Cave in Siberia. Nature 468:1053-1060.

Reinhardt, J. A., B. Kolaczkowski, C. D. Jones, D. J. Begun, and A. D. Kern. 2014. Parallel geographic variation in Drosophila melanogaster. Genetics 197:361-373.

Rheindt, F. E., Fujita, M. K., Wilton, P. R., \& Edwards, S. V. (2014). Introgression and phenotypic assimilation in zimmerius flycatchers (Tyrannidae): Population genetic and phylogenetic inferences from genome-wide SNPs. Systematic Biology, 63(2), 134-152. doi:10.1093/sysbio/syt070

Ribeiro, A. M., P. Lloyd, and R. C. K. Bowie. 2011. A tight balance between natural selection and gene flow in a Southern African arid-zone endemic bird. Evolution 65:3499-3514.

Riento, K., and A. J. Ridley. 2003. Rocks: Multifunctional kinases in cell behaviour. Nature Reviews Molecular Cell Biology 4:446-456.

Roesti, M., S. Gavrilets, A. P. Hendry, W. Salzburger, and D. Berner. 2014. The genomic signature of parallel adaptation from shared genetic variation. Mol Ecol 23:3944-3956.

Romiguier, J., P. Gayral, M. Ballenghien, A. Bernard, V. Cahais, A. Chenuil, Y. Chiari, R. Dernat, L. Duret, N. Faivre, E. Loire, J. M. Lourenco, B. Nabholz, C. Roux, G. Tsagkogeorga, A. A. T. Weber, L. A. Weinert, K. Belkhir, N. Bierne, S. Glemin, and N. Galtier. 2014. Comparative population genomics in animals uncovers the determinants of genetic diversity. Nature 515:261-U243.

Santure, A. W., I. De Cauwer, M. R. Robinson, J. Poissant, B. C. Sheldon, and J. Slate. 2013. Genomic dissection of variation in clutch size and egg mass in a wild great tit (Parus major) population. Mol Ecol 22:3949-3962.

Schielzeth, H., and A. Husby. 2014. Challenges and prospects in genome-wide quantitative trait loci mapping of standing genetic variation in natural populations. Year in Evolutionary Biology 1320:35-57.

Seasholtz, T. M., J. Wessel, F. W. Rao, B. K. Rana, S. Khandrika, B. P. Kennedy, E. O. Lillie, M. G. Ziegler, D. W. Smith, N. J. Schork, J. H. Brown, and D. T. O'Connor. 2006. Rho kinase polymorphism influences blood pressure and systemic vascular resistance in human twins - Role of heredity. Hypertension 47:937-947. 
1055

1056

1057

1058

1059

1060

1061

1062

1063

1064

1065

1066

1067

1068

1069

1070

1071

1072

1073

1074

1075

1076

1077

1078

1079

1080

1081

1082

1083

1084

1085

1086

1087

1088

1089

1090

1091

1092

1093

1094

1095

1096

1097

1098

1099

1100

Singham, G. V., E. L. Vargo, W. Booth, A. S. Othman, and C. Y. Lee. 2012. Polymorphic Microsatellite Loci From an Indigenous Asian Fungus-Growing Termite, Macrotermes gilvus (Blattodea: Termitidae) and Cross Amplification in Related Taxa. Environ Entomol 41:426-431.

Slate, J., and J. M. Pemberton. 2007. Admixture and patterns of linkage disequilibrium in a freeliving vertebrate population. J Evol Biol 20:1415-1427.

Slatkin, M. 2008. Linkage disequilibrium--understanding the evolutionary past and mapping the medical future. Nat Rev Genet 9:477-485.

Smith, B. T., M. G. Harvey, B. C. Faircloth, T. C. Glenn, and R. T. Brumfield. 2014. Target capture and massively parallel sequencing of ultraconserved elements for comparative studies at shallow evolutionary time scales. Syst Biol 63:83-95.

Smukowski, C. S., and M. A. Noor. 2011. Recombination rate variation in closely related species. Heredity (Edinb) 107:496-508.

Stinchcombe, J. R., and H. E. Hoekstra. 2008. Combining population genomics and quantitative genetics: finding the genes underlying ecologically important traits. Heredity 100:158170 .

Stoneking, M., and J. Krause. 2011. Learning about human population history from ancient and modern genomes. Nat Rev Genet 12:603-614.

Sun, J. X., J. C. Mullikin, N. Patterson, and D. E. Reich. 2009. Microsatellites are molecular clocks that support accurate inferences about history. Mol. Biol. Evol. 26:1017-1027.

Sureshkumar, S., M. Todesco, K. Schneeberger, R. Harilal, S. Balasubramanian, and D. Weigel. 2009. A Genetic Defect Caused by a Triplet Repeat Expansion in Arabidopsis thaliana. Science 323:1060-1063.

Taguchi, M., Y. Shigenobu, M. Ohkubo, T. Yanagimoto, T. Sugaya, Y. Nakamura, K. Saitoh, and K. Yokawa. 2013. Characterization of 12 polymorphic microsatellite DNA loci in the blue shark, Prionace glauca, isolated by next generation sequencing approach. Conservation Genetics Resources 5:117-119.

Tautz, D., and M. Renz. 1984. Simple Sequences Are Ubiquitous Repetitive Components of Eukaryotic Genomes. Nucleic Acids Res 12:4127-4138.

Templeton, A. R. 2010. Coalescent-based, maximum likelihood inference in phylogeography. Mol Ecol 19:431-435.

Tiffin, P., \& Ross-Ibarra, J. 2014. Advances and limits of using population genetics to understand local adaptation. Trends in Ecology \& Evolution, 29(12), 673-680. doi:10.1016/j.tree.2014.10.004

Tremblay, D. C., G. Alexander, S. Moseley, and B. P. Chadwick. 2010. Expression, tandem repeat copy number variation and stability of four macrosatellite arrays in the human genome. Bmc Genomics 11:632.

Turner, T. L., and M. W. Hahn. 2010. Genomic islands of speciation or genomic islands and speciation? Mol Ecol 19:848-850.

Uy, J. A. C., R. G. Moyle, C. E. Filardi, and Z. A. Cheviron. 2009. Difference in Plumage Color Used in Species Recognition between Incipient Species Is Linked to a Single Amino Acid Substitution in the Melanocortin-1 Receptor. Am Nat 174:244-254.

Wallberg, A., F. Han, G. Wellhagen, B. Dahle, M. Kawata, N. Haddad, Z. L. P. Simoes, M. H. Allsopp, I. Kandemir, P. De la Rua, C. W. Pirk, and M. T. Webster. 2014. A worldwide survey of genome sequence variation provides insight into the evolutionary history of the honeybee Apis mellifera. Nat Genet 46:1081-1088. 
1101 Weber, C., B. Nabholz, J. Romiguier, and H. Ellegren. 2014. Kr/Kc but not dN/dS correlates positively with body mass in birds, raising implications for inferring lineage-specific selection. Genome Biol 15:542.

Yukilevich, R., T. L. Turner, F. Aoki, S. V. Nuzhdin, and J. R. True. 2010. Patterns and Processes of Genome-Wide Divergence Between North American and African Drosophila melanogaster. Genetics 186:219-239. sparrow Melospiza melodia as a case study. J Avian Biol 41:1-7.

Zink, R. M., and G. F. Barrowclough. 2008. Mitochondrial DNA under siege in avian phylogeography. Mol Ecol 17:2107-2121.

Zweier, C., M. M. Peippo, J. Hoyer, S. Sousa, A. Bottani, J. Clayton-Smith, W. Reardon, J. Saraiva, A. Cabral, I. Gohring, K. Devriendt, T. de Ravel, E. K. Bijlsma, R. C. M. Hennekam, A. Orrico, M. Cohen, A. Dreweke, A. Reis, P. Nurnberg, and A. Rauch. 2007. Haploinsufficiency of TCF4 causes syndromal mental retardation with intermittent hyperventilation (Pitt-Hopkins syndrome). American Journal of Human Genetics 80:9941001. 
1121 Figure 1. Trends in the use of molecular markers over time from a survey of articles on

1122 phylogeography from the journal Molecular Ecology. (A) Plot of the number of articles from

11231992 to 2013 using organelle DNA markers, microsatellites, nuclear sequence-based markers,

1124 color coded according to the key at upper left. The category of nuclear sequence-based markers

1125 includes 8 studies in 2013 using RadSeq, sequence capture, or another next-generation

1126 sequencing technique to genotype SNPs; the remainder of nuclear SNP studies used PCR

1127 approaches. Data on articles using nuclear SNPs and organelle markers are taken from Garrick

1128 et al. (2015) and are presented for every three years for easy comparison with that

1129 study. Overall, during the time period sampled there were 280 studies using only SNPs from

1130 mt- or cpDNA, 101 studies using nuclear SNPs, and 97 studies using nuclear

1131 microsatellites. There were 13 studies that included both nuclear SNPs and microsatellites; these

1132 were excluded from the plot. Sixty-three of the microsatellite studies and 79 of the nuclear SNP

1133 studies also included mt- or cpDNA SNPs and are included. (B) Trends in the total number of

1134 articles from Molecular Ecology using nuclear microsatellites sampled every year from 1992 to

1135 2014. These 318 studies include those using other types of data such as mt- or cpDNA SNPs or

1136 nuclear SNPs. The number of pages in the journal Molecular Ecology per year is shown in a

1137 black line as a guage on growth of the journal as a whole. The full list of articles using

1138 microsatellites can be found in the supplementary material.

1139 Figure 2: Advantages and disadvantages of studying species with low or high levels of

1140 linkage disequilibrium (LD) using genome subsampling methods such as Rad-seq to identify loci

1141 under selection. The matrix covers two sets of species: on the Y-axis, those with low or high 
1142 levels of $\mathrm{LD}$, as discussed in the main text. On the $\mathrm{X}$-axis are listed the advantages or

1143 disadvantages of studying such species using the Rad-seq approach. In each cell is a description

1144 of common situations encountered in the search for loci under selection. See text for further

1145 discussion.

1146 Figure 3: Two examples of low- and high linkage disequilibrium species. A) Plot of

1147 pairwise $\mathrm{r}^{2}$ (a measure of linkage disequilibrium) between SNPs across 1500 base pairs of the

1148 HSP90a gene in House Finches, a common North American songbird. SNPs from an Arizona

1149 population are in black, those from an Alabama population in red. This species is characterized

1150 by large population sizes, resulting in a high population recombination rate and low levels of LD.

1151 From Backström et al. (2013). B) Similar plot of pairwise $r^{2}$ and physical distance in kilobases

1152 in various dog breeds and wild populations of gray wolves (Canis lupus). Notice how high levels

1153 of LD extend hundreds of kilobases; those same levels of LD extend only a few hundred bases in

1154 the case of the House Finches. Image from Boyko (2011); see also Boyko et al. (2010). Both

1155 images used under the Creative Commons 2 License

1156 https://creativecommons.org/licenses/by/2.0/.

1157 Figure 4. Correlations of Rad-seq SNP variation and environmental variables across

1158 geographic space in the lizard Anolis carolinensis. A) Environmental associations across

1159 geographic space of each SNP identified by RADseq and the mean temperature of the coldest

1160 quarter of the year at each of 6 localities distributed across the species range, calculated in

1161 Bayenv2 (Gunther and Coop 2013). The horizontal and vertical dotted lines represent 99\%

1162 cutoffs for significance of Pearson correlation and Bayes Factor associations, respectively. Filled

1163 points indicate candidate SNPs falling within the top $1 \%$ of both axes. B) The genomic position 
1164 of each outlier SNP in panel A on the 6 annotated macrochromosomes of the A.carolinensis

1165 genome (Alfoldi et al. 2011).

1166 Figure 5. Estimates of $F_{\mathrm{st}}$ and associated Fisher's p-values for each SNP compared

1167 between pre- (1990) and post- Mycoplasma (2003) epizootic individuals sampled from Auburn,

1168 Alabama. The dotted line on the plot of $\mathrm{p}$-values shows the uncorrected cutoff of $\mathrm{p}=$

1169 0.05. After Bonferroni correction, no SNPs achieve significant $F_{\text {st }}$ (see text). SNP positions are

1170 depicted as mapped to the Zebra Finch genome, assuming synteny between the House Finch and

1171 Zebra Finch genomes. High values of $F_{\mathrm{st}}$ are not always associated with high Fisher's p-values,

1172 usually due to incomplete data matrices and concomitant smaller sample sizes at those positions,

1173 a situation common in RadSeq data. Image of House Finch from

1174 http://www.flickr.com/photos/11652987@N03/7315942062 and used under the Creative

1175 Commons 2 License https://creativecommons.org/licenses/by/2.0/. 
A
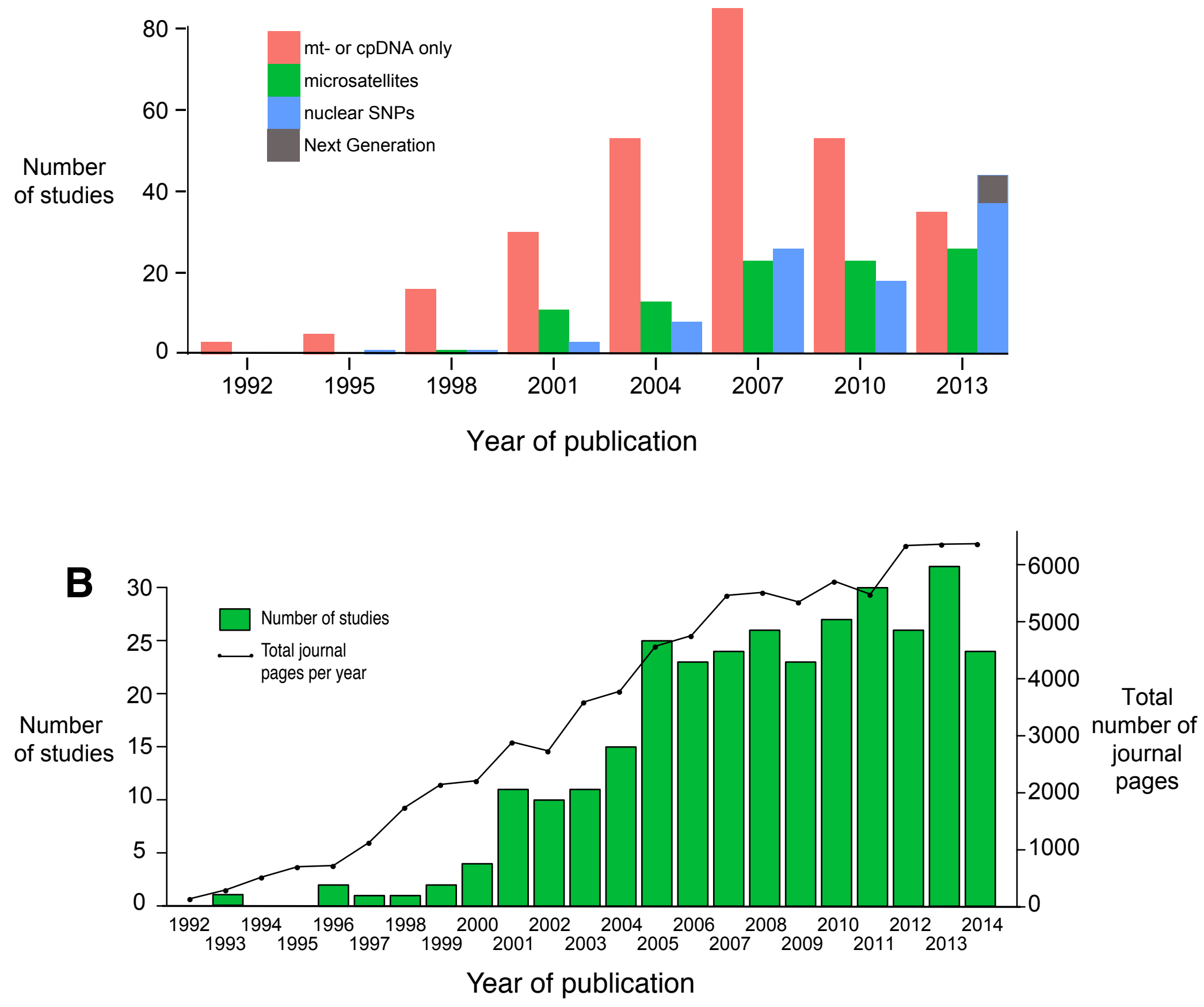
Rad-seq or other genome subsamping method

Advantages Disadvantages

Low LD species

SNPs exhibiting selection

Difficult to find SNPs likely close to linked to causal variants; causal variants many SNPs required

High LD species

\begin{tabular}{|c|c|}
\hline $\begin{array}{c}\text { SNPs exhibiting selection } \\
\text { likely close to } \\
\text { causal variants }\end{array}$ & $\begin{array}{c}\text { Difficult to find SNPs } \\
\text { linked to causal variants; } \\
\text { many SNPs required }\end{array}$ \\
\hline $\begin{array}{c}\text { Easier to find SNPs linked } \\
\text { to causal variants }\end{array}$ & $\begin{array}{c}\text { Long linkage blocks } \\
\text { containing many } \\
\text { candidate genes }\end{array}$ \\
\hline
\end{tabular}

Figure 2 
A
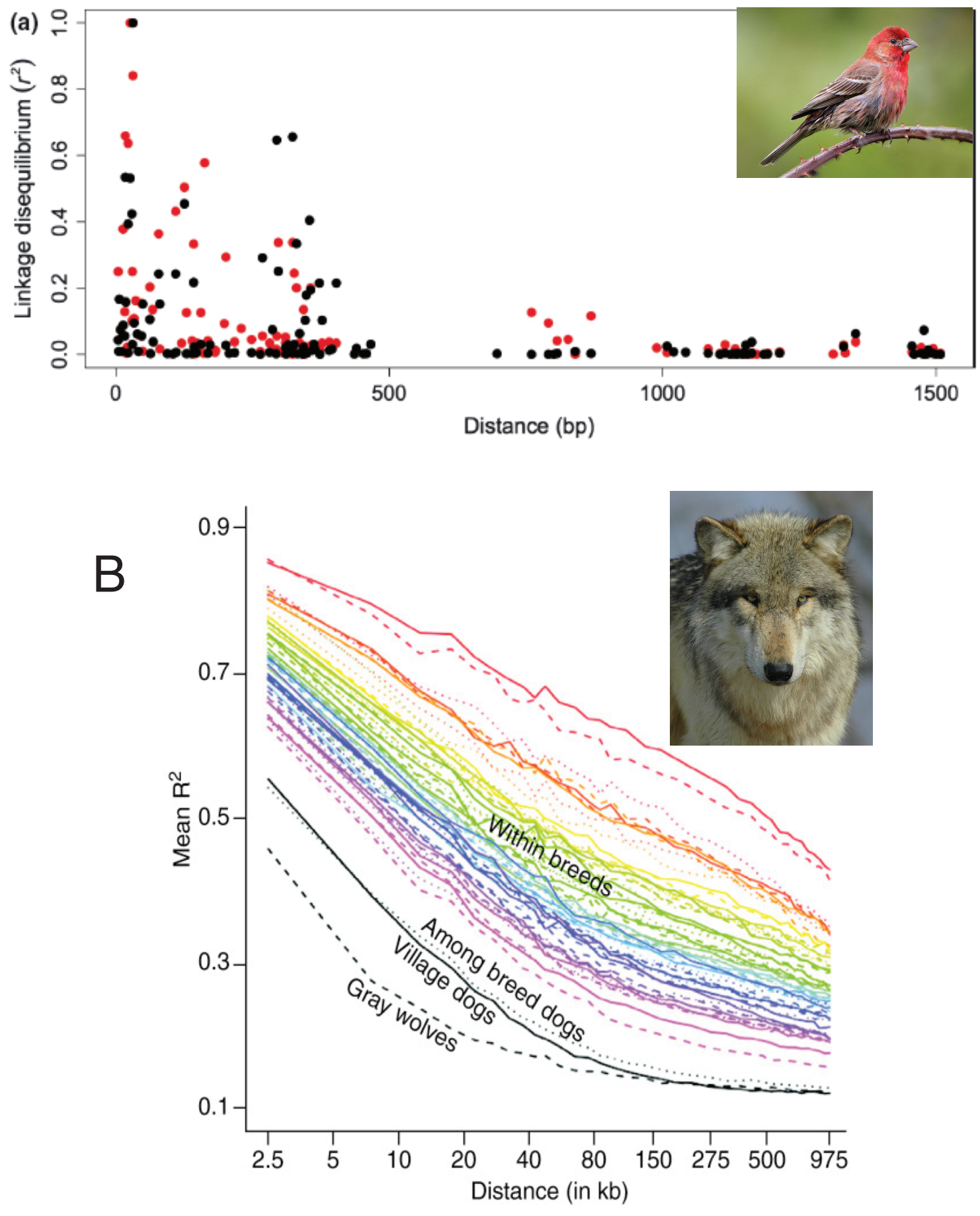

Figure 3 
A

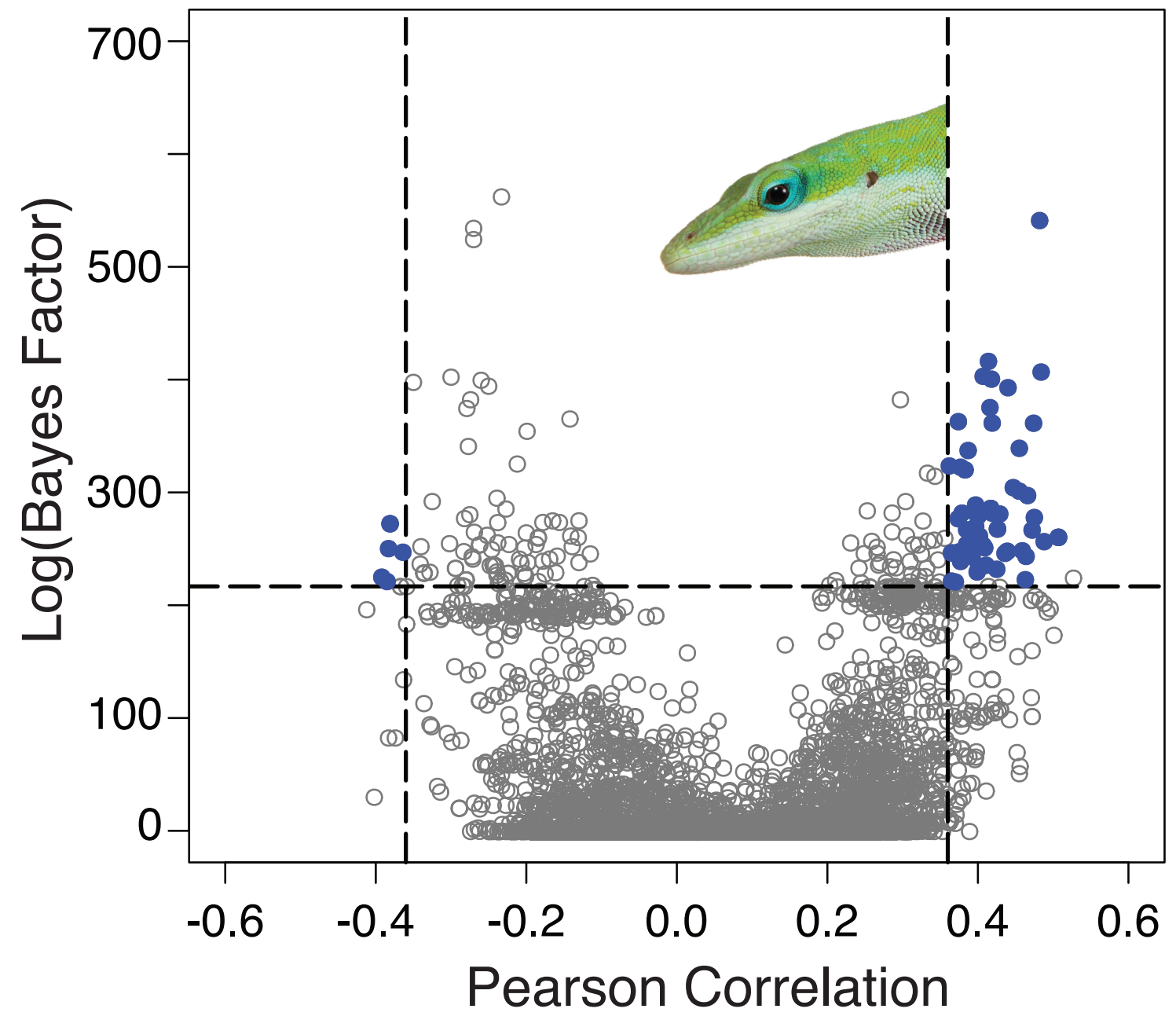

B
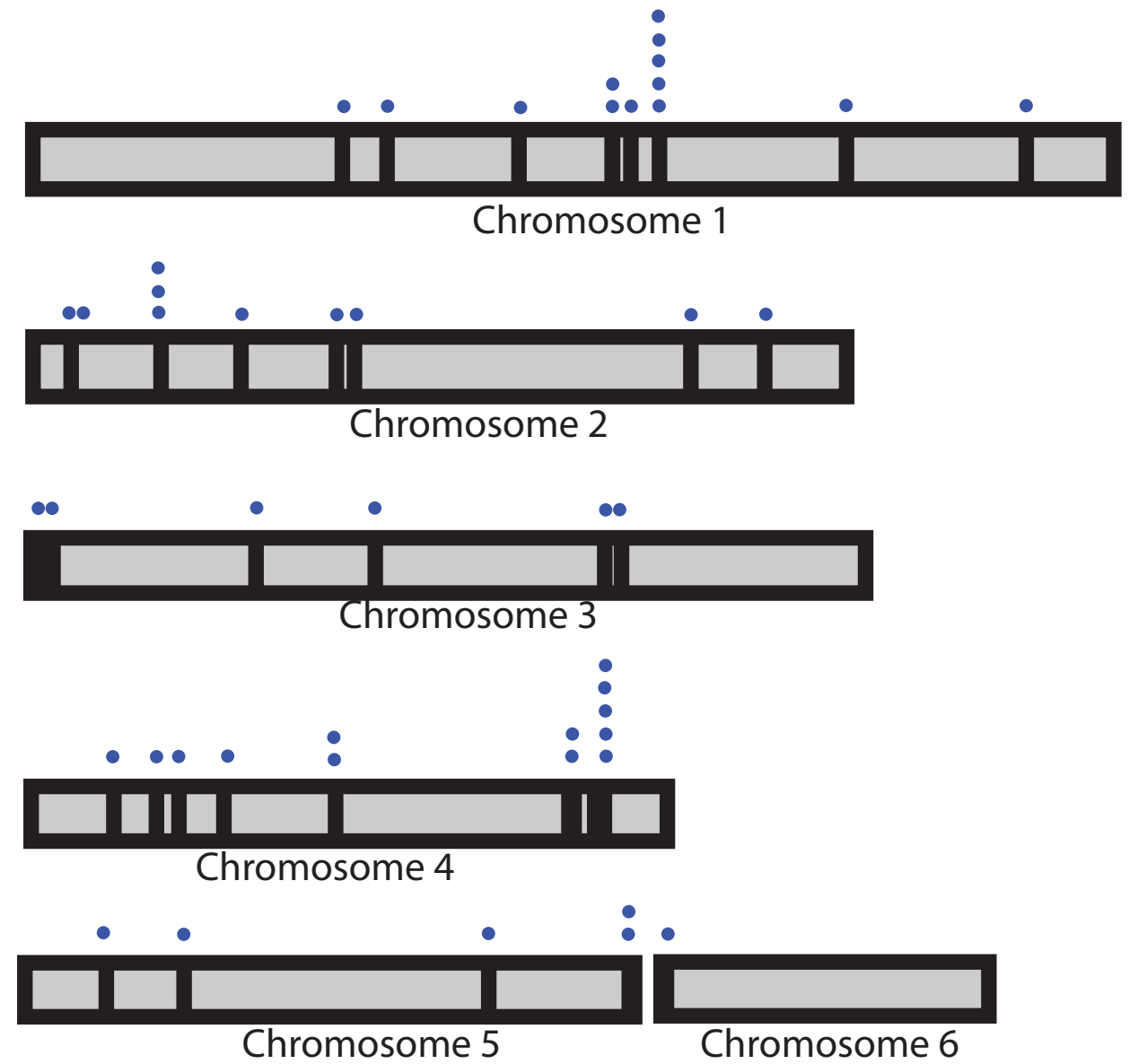

Figure 4 

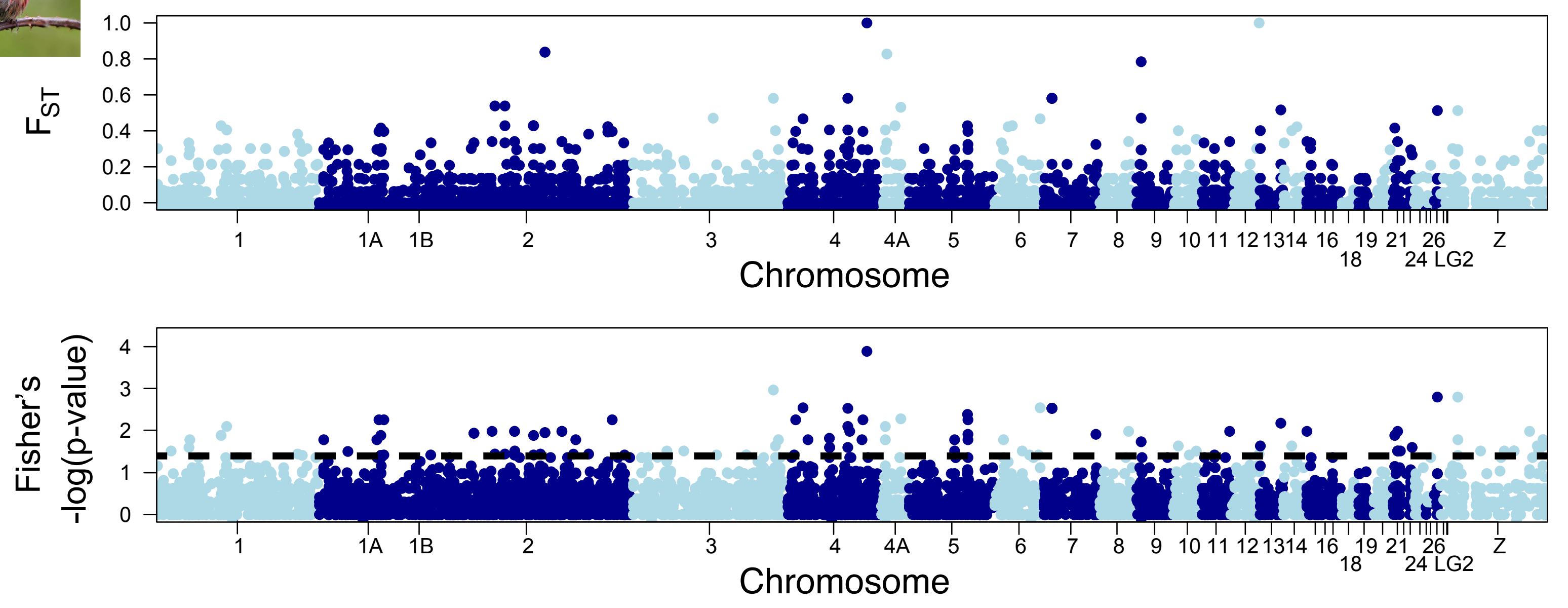

Figure 5 